\title{
Sprachwissenschaft im Fokus
}

Positionsbestimmungen und Perspektiven

Herausgegeben von Ludwig M. Eichinger

\section{DE GRUYTER}


ISBN 978-3-11-035881-0

e-ISBN [PDF] 978-3-11-040159-2

e-ISBN [EPUB] 978-3-11-040166-0

ISSN 0537-7900

\section{Library of Congress Cataloging-in-Publication Data}

A CIP catalog record for this book has been applied for at the Library of Congress.

\section{Bibliografische Information der Deutschen Nationalbibliothek}

Die Deutsche Nationalbibliothek verzeichnet diese Publikation in der Deutschen

Nationalbibliografie; detaillierte bibliografische Daten sind im Internet über http://dnb.dnb.de abrufbar.

(C) 2015 Walter de Gruyter GmbH, Berlin/München/Boston Gesamtherstellung: Hubert \& Co. GmbH \& Co. KG, Göttingen

@ Gedruckt auf säurefreiem Papier

Printed in Germany

www.degruyter.com 


\title{
Die Umparametrisierung der Grammatik durch Literalisierung. Online- und Offlinesyntax in Gegenwart und Geschichte
}

\begin{abstract}
Moderne Grammatiktheorien sind statisch, d.h. skriptizistisch und synchronizistisch. Dies bedeutet, dass deren Beschreibungsapparat auf die Strukturen gegenwärtiger Schrift- und Standardsprachen zugeschnitten ist. Im Beitrag wird für einen dynamischen, d.h. nichtskriptizistischen und nichtsynchronizistischen, Perspektivwechsel in der Grammatikforschung plädiert, der auf folgenden empirisch fundierten Überlegungen basiert:

1. Literalisierung ist eine kulturelle Universalie, die kognitiv verankert ist.

2. Es sind unterschiedliche Phasen der Literalisierung zu unterscheiden.

3. Literalisierung im Allgemeinen und die Phasen der Literalisierung im Besonderen haben Konsequenzen für die grammatische Struktur.

4. Die Interpretation von grammatischen Strukturen ist nur vor der Folie der jeweiligen Phase der Literalisierung möglich.

5. Ein dynamisches Grammatikmodell muss das historische Verhältnis auch begrifflich abbilden. Dies wird an zentralen grammatischen Konzepten wie Aggregation vs. Integration, Wortgruppe vs. Phrase und an der Wortstellung (Verbklammer, Stellungsfeldermodell, Satzrandglieder) veranschaulicht.

6. Historisch ist von einem dynamischen Verhältnis von Online- und Offlinesyntax, von syntaktischer Zeitlichkeit und syntaktischer Räumlichkeit, auszugehen. Was zu einer bestimmten Zeit und in einer bestimmten Varietät als Onlinestruktur zu interpretieren ist, hängt von dem jeweiligen historischen Verhältnis von Online- und Offlinestrukturen ab.
\end{abstract}

\section{Von der statischen zur dynamischen Grammatik}

Der Gegenstand einer Theorie lässt sich auf drei Ebenen untersuchen (zum Nachfolgenden vgl. Ágel 2003):

1. Explizit: was gesagt wird und wohl auch gemeint ist;

2. Implizit: was nicht gemeint ist, aber daraus folgt, was gesagt wird;

3. Ex-silencio: was wohl gemeint ist, aber nur daraus folgt, was nicht gesagt wird. 
Explizite oder Ex-silencio-Gegenstände der Mainstream-Grammatiktheorien des 20. Jahrhunderts sind das gesprochene oder das modal indifferente Wort. Implizit sind sie allerdings alle skriptizistisch, was als „scriptist bias of modern linguistics“ (Harris 1980, S. 8) oder als „The Written Language Bias in Linguistics“ (Linell 2005) bekannt ist.

Neben diesem skriptizistischen Erbe lastet auf der Grammatikforschung auch ein synchronizistisches Erbe, das, kurz gesagt, darin besteht, dass der Beschreibungsapparat moderner Grammatiktheorien auf die Strukturen gegenwärtiger Standardsprachen zugeschnitten ist. Folglich ist im 20. Jahrhundert die gesamte historische Grammatikforschung ohne gegenstandsadäquate theoretische Überdachung geblieben. Die historische Grammatikforschung wird also immer noch als eine Art Angewandter Sprachwissenschaft betrieben: Gegenwartsbezogen mehr oder weniger bewährte Konzepte wie Phrase, Satz, Klammerstruktur, Stellungsfelder u.v.a. werden theoretisch mehr oder weniger reflektiert und methodisch mehr oder weniger kontrolliert auf ältere Sprachstufen oder auf den Grammatikwandel angewandt. Die Frage, ob sie überhaupt gegenstandsadäquat sind, stellt sich in aller Regel nicht. Aber es geht nicht nur um die historische Grammatikforschung. Wenn moderne Grammatikmodelle skriptizistisch und synchronizistisch - kurz: statisch - sind, betrifft das Problem die gesamte Grammatikforschung. ${ }^{1}$

Ich werde im Folgenden zu zeigen versuchen, dass es ausreichende empirische Evidenz für einen dynamischen, d.h. nichtskriptizistischen und nichtsynchronizistischen, Perspektivwechsel in der Grammatikforschung gibt. ${ }^{2}$ Dabei wird das Augenmerk auf die Syntax gerichtet werden.

1 Als Fazit seiner Kritik an meinem Synchronizismus-Postulat schreibt Peter Eisenberg (2007, S. 293): „Die Frage nach dem Zustand und die Frage nach seiner Genese bleiben zwei Paar Schuhe." Das ist richtig. Folglich sollte man mit Theorien, die sich auf Zustandsbeschreibungen spezialisiert haben, vorsichtig sein, wenn es um die Modellierung der Genese geht. Aus der genuinen Historizität von Sprache (Coseriu 1974) folgt sogar, dass Sprach- und Grammatiktheorien nur als integrierte Bestandteile von Sprach- und Grammatikwandeltheorien zu begreifen sind. Der Synchronizismus hatte und hat verheerende Konsequenzen: An vielen Universitäten wurden die Lehrstühle für Sprachgeschichte/Historische Linguistik geschlossen bzw. in Lehrstühle für Theoretische, sprich: Nichthistorische, Linguistik verwandelt. Ganze Grammatiker-Generationen wuchsen und wachsen immer noch ohne sprachhistorische Ausbildung auf, was wiederum die synchronizistische Theoriebildung konserviert. Weil sie akademische Mainstream-Lehre ist, erscheint sie sogar plausibel.

2 Als den dritten - variationslinguistischen - Pfeiler einer dynamischen Grammatik sehe ich das Sprachdynamik-Konzept von Schmidt/Herrgen (2011) an. Schmidt und Herrgen bekämpfen nämlich ein drittes Erbe des 20. Jahrhunderts, das man Varietätenrealismus nennen könnte: „Setzt man Varietäten [...] als homogene Subsysteme an, so stellt dies erneut nur methodisch eine bessere Annäherung an die heterogene Gesamtsprache dar. Es bedeutet aber theoretisch, 


\section{Literalisierung und Grammatik}

\subsection{Literalisierung und Kognition}

Mit Universalität werden in der Grammatikforschung in der Regel Universalien im sprachtypologischen Sinne oder das Postulat einer Universalgrammatik assoziiert. Aus evolutionärer Perspektive sind allerdings alle Phänomene universal, die nicht kontingent sind, d.h., die unter bestimmten biologischen, kognitiven oder kulturellen Bedingungen notwendigerweise auf den Plan treten oder, wenn sie schon auf dem Plan sind, vergleichbare Merkmale und Entwicklungen aufweisen. ${ }^{3}$ In diesem Sinne ist Literalisierung (auch) ein universelles Phänomen mit universalen Merkmalen und Entwicklungszügen. Wie sich ihre kulturelle Universalität in Biologie und Kognition manifestiert, soll kurz angedeutet werden:

Das Thema ,Mündlichkeit und Schriftlichkeit‘ erfreut sich in den letzten Jahren zunehmender Aufmerksamkeit auch in medizinischen und psychologischen Fachzeitschriften. Beispielsweise kommen Aaron/Joshi (2006), die die biolinguistische Forschung aus diversen sprachevolutionären Perspektiven Revue passieren lassen, zum Schluss, dass geschriebene Sprache genauso natürlich sei wie gesprochene Sprache, beide seien relativ unabhängige Produkte einer biologischen und sozio-kulturellen Evolution.

Was den Kompetenzaspekt anbelangt, ist Sprache bei Illiteraten in der dominanten Gehirnhälfte nicht gut verankert, während es bei Literaten eine ausgeprägte Links/Rechts-Asymmetrie gibt. Dies wurde auch durch vergleichende Forschungen zu Illiteraten und Literaten mit LH- und RH-Läsionen bestätigt (Parente/Fonseca/Scherer 2008). Zu diesem Befund passt auch das neurolinguistische Konzept der propositionalen vs. nonpropositionalen Sprachkompetenz von Van Lancker (1987, S. 55 ff.). Nach ihr ist die nonpropositionale Sprachkompetenz kohäsiv, d.h. analog, die propositionale dagegen analytisch, d.h. digital. Erstere sei entweder in beiden Gehirnhälften oder in der rechten Hemisphäre lokalisierbar. Demgegenüber sei Letztere ausschließlich auf die linke Gehirnhälfte spezialisiert.

durch die Vervielfältigung des Gegenstandsinadäquaten (d.h. der homogenen Kompetenz) einen in der Realität anders strukturierten Gegenstand mit einem ungeeigneten Konzept erfassen zu wollen.“ (Schmidt/Herrgen 2011, S. 21).

3 Vgl. auch die Forschungsrichtung der sogenannten Ethnosyntax: ,broadly defined as the study of connections between the cultural knowledge, attitudes, and practices of speakers, and the morphosyntactic resources they employ in speech“ (Enfield 2002, S. 3). 
Diese Befunde stellen eine späte Bestätigung der großangelegten kulturhistorischen Studie des Psychologen Julian Jaynes (1993) dar, der die Herausbildung der Links/Rechts-Asymmetrie - das sogenannte Verstummen der bikameralen Psyche - mit der Literalisierung in Verbindung gebracht hat.

Überzeugende empirische Evidenz für literalisierungsbedingte Kompetenzunterschiede in der deutschen Gegenwartsgrammatik liefert die empirische Studie von Jürgen Erich Schmidt (1993) zur sogenannten Attribuierungskomplikation.

Das kognitionswissenschaftliche Fundament für eine dynamische Grammatik bildet Eckart Scheerers Theorie, nach der die Literalisierung zur Umgestaltung des kognitiven Systems geführt hat (Scheerer 1993). Laut Scheerer schließen sich die symbolorientierte und die konnektionistische, d.h. netzwerkbasierte, Perspektive auf die Funktionsweise mentaler Prozesse nicht aus, da biologische Systeme „keine symbolische Funktionsebene“ haben, aber „den Gebrauch von Symbolen erwerben können - vorausgesetzt, sie werden mit externen Symbolsystemen und dem Umgang mit ihnen konfrontiert“ (ebd., S. 162). Die Fähigkeit zur Symbolmanipulation sei eine neue Funktion des konnektionistischen kognitiven Systems, die erst historisch, im Zuge der Literalisierung, erworben wurde.

Aus dieser Auffassung folgen wichtige Schlüsse (siehe auch Ágel 1999; 2003):

1. Da literales Denken erst historisch erworben wird, setzt der Erwerb der Fähigkeit zur Symbolmanipulation das netzwerkbasierte Denken voraus, aber nicht umgekehrt. Literalisierung führt also zu neuen Denkmustern. Das sind die berühmten Folgen der Schriftkultur, wie sie vor allem von Havelock (1963), Goody (1977; 1987) und Ong (1987) herausgearbeitet wurden.

2. Die neuen symbolorientierten Denkmuster verdrängen nicht notwendigerweise die alten netzwerkorientierten, sondern sie überlagern sie. Es kommt zur Vertikalisierung von oral-literalen Denkmustern.

3. Literalisierung bleibt nicht ohne grammatische Konsequenzen. Der Erwerb der Fähigkeit zur Symbolmanipulation verändert grundlegend unsere gesamte Wahrnehmung von sprachlichen Tätigkeiten und Produkten. Dies bedeutet sprachwandeltheoretisch, dass aggregative grammatische Strukturen zunehmend von integrativen verdrängt oder überlagert werden (vgl. Abschnitt 2.2).

Trotz zahlreicher Untersuchungen zum oral-konnektionistischen Denken ist es für uns literal-symbolorientiert Sozialisierten äußerst schwer, vielleicht sogar unmöglich, das oral-konnektionistische Denken nachzuvollziehen. ${ }^{4}$ Trotzdem

4 Die immer noch beste und aufschlussreichste Annäherung ist Malinowskis (1974) „Das Problem der Bedeutung in primitiven Sprachen“. 
möchte ich auf ein grammatisches Merkmal der Sprache der Pirahã eingehen, das eine überraschende Parallele zum Ahd. ermöglicht. ${ }^{5}$

Die Pirahã-Kultur basiert nach Everett auf dem Prinzip der Unmittelbarkeit der Erfahrung:

Im Pirahã gibt es nur deklarative Äußerungen, die sich direkt auf den Augenblick des Sprechens und nur auf Sachverhalte beziehen, die entweder selbst erlebt oder durch einen lebenden Augenzeugen bestätigt wurden. In der Kultur der Pirahã muss alles „rückversichert“ werden. Es muss selbst erlebt, von dritter Seite bezeugt oder aus Hinweisen gefolgert sein. Darüber hinaus tragen Verben im Pirahã ein Suffix, das die Nachweisquelle angibt: xáagahá = „beobachtet“, hiai = „gehört“ und sibiga $=$,gefolgert“. (Everett 2013, S. 384 f.)

(1a) Kóxoí higáísai. Kohoi hi goó gáísai. Xaogií goó gáísai. Báaxaí tíi.

Kóxoí sagte das. Kohoi sagte das. Ausländische Frau sagte das. Ich bin schön. (Everett 2013, S. 391)

(1b) Kóxoí sagte, dass Kohoi sagte, dass die ausländische Frau sagte, dass ich schön bin. (Übersetzung V.Á.)

Im Pirahã muss und kann nur der verbale Zentralknoten mit einem Evidenzmarker authentifiziert werden. Würde man einen verbalen Zentralknoten translatieren, d.h. rekursiv einsetzen, könnte er nicht mehr authentifiziert werden (Everett 2013, S. 385f.). Im Pirahã sind also wegen der obligatorischen Markierung der Evidentialität am verbalen Zentralknoten weder eingebettete Nebensätze noch komplexe Nominalisierungen möglich. Pirahã können also aus kulturellen Gründen - Prinzip der Unmittelbarkeit der Erfahrung - keine translatierten Knoten authentifizieren. Daraus lässt sich eine Art Kultur-GrammatikPfad herleiten:

(2) Unmittelbarkeit der Erfahrung $\rightarrow$ Erzählen $\rightarrow$ Authentifizierung des Erzählten $\rightarrow$ Evidenzmarker nur am zentralen Verbalknoten $\rightarrow$ Parataxe

5 Pirahã ist die letzte noch existierende Mura-Sprache im Amazonasgebiet mit ca. 360 Sprechern. Es waren die Arbeiten von Everett (2005; 2010; 2013), die der Kultur und der Sprache der Pirahã Weltruhm eingebracht haben - der Sprache vor allem deshalb, weil die Pirahã-Grammatik nicht rekursiv ist (siehe auch das Beispiel (1a)), was die Annahme einer Universalgrammatik grundlegend in Frage stellt. Zur Rekursivität siehe Evans/Levinson (2009, S. 442 ff.). 
Parataktische Organisation des Diskurses muss also keinesfalls ein inhärentes Merkmal primärer Oralität sein, sie kann auch eine Folge obligatorischer und nichtrekursiver Evidenzmarkierung sein. ${ }^{6}$

Hier gibt es überraschende Parallelen zum Ahd. (Belege (3) bis (5) aus dem Hildebrandslied, (6) aus dem Muspili, zit. n. Kotin/Schönherr 2012, S. 403 f.):

(3) 1: Ik gihôrta đat seggen

(4) 15-16: dat sagetun mi usere liuti, / alte anti frote, dea erhina warun

(5) 42-43: dat sagetun mi seolidante / westar ubar wentilseo

(6) 37: daz hortih rahhôn dia uueroltrehtuuison ,das habe ich die Frommen dieser Welt sagen hören ...

Auch hier geht es um die Authentifizierung des Erzählten:

Geht man davon aus, dass die overt kodierte Evidentialität eine spezifische Form der „Garantie für die Wahrheit“ des Dargestellten war und somit die Konsistenz der konzeptionell mündlichen (wenn auch später medial schriftlichen) Überlieferung bewerkstelligte, muss man zugeben, dass die EV-Signale [= evidentiellen Signale, V.Á.] in diesem System statusmäßig sehr nahe zu denen in den Sprachen mit grammatisch kodierter Evidentialität stehen. (Kotin/Schönherr 2012, S. 404)

Und wenn, wie in Otfrids Evangelienbuch, evidentielle mit epistemischen Markern kombiniert werden, gibt es eine deutliche Hierarchie zugunsten der Evidentialität (Kotin/Schönherr 2012, S. 409).

Diese Hierarchie ist typisch für die erste Phase der Literalisierung, die Verschriftung. ${ }^{7}$ In der Phase der Verschriftung verdrängt oder überlagert das literale Darstellen das orale Erzählen noch nicht. ${ }^{8}$ Untersuchungen zur Epistemifizierung zeigen (siehe Ágel 1999 mit weiterer Literatur), dass die Epistemifizierung im großen Stil erst in der zweiten Phase der Literalisierung, der Verschriftlichung, einsetzt. In dieser Phase, im Deutschen ab dem 16. Jahrhundert, ist mit einer sukzessiven Umkehrung der altdeutschen Hierarchie zu rechnen:

\footnotetext{
6 Parataxe hat auch andere Quellen, z.B. die Redundanz mündlichen Erzählens.

7 ,Verschriftung“ ist nach Koch und Oesterreicher der mediale, ,Verschriftlichung der konzeptionelle Aspekt der Literalisierung (Koch/Oesterreicher 1994, S. 587).

8 Im Erzählen gibt es im Gegensatz zum Darstellen auch keinen Unterschied zwischen direkter und indirekter Redewiedergabe. Entscheidend ist die Einheit von Erzählung und Erzähler: Eine Erzählung ist keine Kette von Propositionen, über die der Erzähler urteilt oder zu denen er Einstellungen hat, sondern er ist Teil der Erzählung.
} 


\author{
Oralität: \\ Erzählen $\rightarrow$ Authentifizierung des Erzählten $\rightarrow$ Evidenzmarker \\ Literalität: \\ Darstellen $\rightarrow$ Einstellung (,Meinung`) des Darstellenden zum Dargestellten \\ $\rightarrow$ epistemische Marker \\ Literalisierung: \\ Darstellen verdrängt/überlagert Erzählen \\ Einstellung verdrängt/überlagert Authentifizierung \\ Epistemizität verdrängt/überlagert Evidentialität
}

\title{
2.2 Die Parameter ,Aggregation' und ,Integration`
}

Die grammatischen Korrelate der Literalisierung sind im Spannungsfeld von zwei übergreifenden Prinzipien der grammatischen Organisation - dem Kontextgrammatischen und dem Symbolgrammatischen Prinzip - zu lokalisieren. ${ }^{9}$ In enger Anlehnung an Arbeiten von Wilhelm Köller zur Perspektivität im Allgemeinen und in der Grammatik im Besonderen (siehe zuletzt Köller 2004) wurde dabei der Aggregationsparameter als der Kernbereich des Kontextgrammatischen Prinzips und der Integrationsparameter als der Kernbereich des Symbolgrammatischen Prinzips identifiziert. ${ }^{10}$

Aggregativität soll nun an den Beispieltypen (8) bis (12), deren integrative Pendants die entsprechenden schriftsprachlichen Strukturen des Gegenwartsdeutschen sind, illustriert werden: ${ }^{11}$

9 Damit sind solche Typen von Strukturierungsunterschieden gemeint wie z.B. Situationsgebundenheit vs. Abstraktheit, Analogizität (Dichte) vs. Digitalität (Diskretheit), Ausdrucksbezogenheit vs. Wortbezogenheit, Lose Fügung vs. Kompaktheit, Formale Inkonstanz vs. formale Konstanz, Polyfunktionalität vs. Funktionale Eindeutigkeit, Varianz vs. Invarianz, Formelhaftigkeit vs. Propositionalität, Inhalts- vs. Formorientiertheit, Tätigkeits- vs. Produktbezogenheit, Gebrauch vs. Bedeutung (siehe Ágel 2003; 2007 und 2010b mit weiterer Literatur). Die Unterscheidung von ,Kontextgrammatik und ,Symbolgrammatik‘ stammt, in Anlehnung an Scheerer (1993), von Eisenberg (1995).

$10 \mathrm{Zu}$,Aggregation vs. Integration“ vgl. auch Raible (1992) und Koch/Oesterreicher (2011, S. $12 \mathrm{f}$. und 99). Empirisch nachgewiesen werden konnte die Relevanz von diesen Parametern für die nhd. Grammatik im Bereich von Nähe und Distanz (Ágel/Hennig (Hg.) 2006) und der expliziten und elliptischen Junktion (siehe KAJUK; Ágel 2010a; Ágel/Diegelmann 2010; Hennig 2010a, b). 11 Die mit römischen Ziffern von I bis VII zitierten Texte entstammen einem im Aufbau befindlichen Nähekorpus des Nhd. Dabei wurde der Zeitraum 1650-2000 in sieben Abschnitte à 50 Jahre ( $\mathrm{I}=1650-1700$; II = 1700-1750 ... VII = 1950-2000) eingeteilt. Der jeweilige Entstehungs- 
(8) Dan es ist verbodten, kein geladten Rohr in dißem Walt zu tragen ... (Güntzer I, S. 41 r)

(9) Beynöben hatte ich auch 12 fl., so ich zusamengelegt hab, waß $\boldsymbol{\beta}_{\text {nom }} \mathbf{m i h r}_{\text {dat }}$ $\mathrm{zu}$ Zeitten von meinen Frindten ist vererdt worden undt [waß $\mathrm{akk}_{\mathrm{a}}$ ] $\left[\mathrm{ich}_{\mathrm{nom}}\right.$ ] mitt Zinstechen verdienet habe. (Güntzer I, S. 40 v)

(10) Itzt avanzierten wir bis unter die Kanonen, wo wir mit dem ersten Treffen abwechseln mußten. (Bräker III, S. LV)

(11) Das Hanaw war belägert von kaiserischem Volk und war besetzet mit Schweden. (Bauernleben I, S. 35)

(12) welche du an mihr armer Sindter bewißen hast (Güntzer I, S. 66 r)

Beim Typus (8) handelt es sich um syntaktische Subordination. Dieser entspricht jedoch keine semantische Subordination: Die Setzung der Negation in der Infinitivkonstruktion ist von der Realisierung des negativ-implikativen Matrixverbs verbieten unabhängig. ${ }^{12}$

Typus (9) ist doppelt aggregativ. Im zweiten Nebensatz wird einerseits kein Akkusativobjekt waß realisiert, obwohl waß im ersten Nebensatz im Nominativ steht. Andererseits wird kein Subjektsnominativ (ich) realisiert, obwohl das Bezugspronomen im Dativ steht (mihr). Es handelt sich um „kategoriale aggregative Koordinationsellipsen“ (Hennig 2010a, b). ${ }^{13}$

Typus (10) ist ein Beleg für die westoberdeutsche Relativpartikel wo aus dem 18. Jahrhundert. Im heutigen Standarddeutsch wäre hier nur das Relativpronomen möglich. ${ }^{14}$

Typus (11) ist deshalb besonders aufschlussreich, weil er unauffällig ist. Er ist aggregativ, weil die Kopula auch im zweiten Konjunkt realisiert wird, was die Integration der beiden Konjunkte schwächt.

(12) ist schließlich eine Apposition ohne Kasuskongruenz.

Aggregative Strukturen wie (8) bis (12) ist gemeinsam, dass die Regeln der syntaktischen Konstruktionsbildung weniger formal sind als im heutigen Standard. Im 17. Jahrhundert sind sie noch quer durch alle Textsorten belegt. Ab dem 18. Jahrhundert kommen sie jedoch zunehmend nur noch in Nähetexten vor. Warum?

abschnitt ist den Zitierformen zu entnehmen. „Güntzer I“ ist beispielsweise ein Nähetext aus der Zeit zwischen 1650 und 1700.

12 Die Infinitivkonstruktion bei Güntzer ist also assertiert. Im Gegensatz dazu wäre die Proposition der entsprechenden Infinitivkonstruktion im Gegenwartsdeutschen präsupponiert und die Setzung des Negationsartikels nicht möglich.

13 Die in [...] gesetzten Sequenzen in (9) stellen keine im Text vorkommenden Sprachzeichen dar, sondern lediglich die Rekonstruktionen von kategorialen aggregativen Koordinationsellipsen.

14 Zur Erklärung des Sprachwandels im Bereich der Relativa siehe Ágel (2010b). 


\subsection{Vertikalisierung des Varietätenspektrums}

Das Schlüsselkonzept ist Oskar Reichmanns Theorie der Vertikalisierung des Varietätenspektrums (1988, 1990 und 2003). Reichmann (2003, S. 38 ff.) unterscheidet insgesamt sechs Vertikalisierungsdimensionen, von denen hier drei relevant sind: ${ }^{15}$

1. Sprachsoziologische Vertikalisierung: Die horizontal-polyzentrische Varietätenorganisation weicht einer vertikal-unizentrischen, die sich an der Prestigevarietät, d.h. der Leitvarietät, orientiert. Während Sprachwandel bis ins 15. Jahrhundert vornehmlich im geografischen Raum stattfand, findet $a b$ dem 16. Jahrhundert kaum mehr großflächiger Sprachwandel statt, da durch die sprachsoziologische Umorientierung die horizontalen Varietätenkontakte geschwächt wurden.

2. Mediale Vertikalisierung: Hier geht es um die Nähe-Distanz-Dimension, denn Vertikalisierung ist auch „eine Entwicklung aus der [...] konzeptionellen Mündlichkeit heraus in die konzeptionelle Schriftlichkeit als sprachkulturelles Orientierungszentrum hinein“" (ebd., S. 42).

3. Strukturelle Vertikalisierung: Vertikalisierung als Strukturwandel wird von Reichmann als Folge der medialen Vertikalisierung beschrieben. Reichmanns lange Liste aus der Syntaxgeschichte der nhd. Schriftsprache (Reichmann 2003, S. 47) enthält zum Großteil Phänomene, die einer integrativen Umparametrisierung der Leitvarietät zuzuordnen sind.

Die Vertikalisierung hat Konsequenzen für die varietätenbezogene Funktionalisierung von Aggregation und Integration:

- Das ursprüngliche Nebeneinander von aggregativen und integrativen Strukturen verwandelt sich zunehmend in ein Übereinander: Maximal integrativ ist die Leitvarietät, maximal aggregativ sind die Dialekte. Entsprechend ist das Prestige von integrativen Strukturoptionen hoch und von aggregativen niedrig.

- Infolgedessen werden aggregative Strukturen ganz aufgegeben oder beschränken sich auf den Substandard.

- Umgekehrt sind die neuen Strukturen, die in der Vertikalisierungszeit entstehen, integrativ und verbreiten sich von oben nach unten.

15 Auf eine vierte, die sprachreflexive (,sprachbewusstseinsgeschichtliche“), Vertikalisierungsdimension komme ich in Abschnitt $4.2 \mathrm{zu}$ sprechen. 
Schematisch lassen sich die Phasen der Literalisierung im Deutschen wie folgt zusammenfassen: ${ }^{16}$

\begin{tabular}{lll}
\hline primäre Literalität & sekundäre Literalität & tertiäre Literalität \\
\hline Verschriftung & Verschriftlichung & „sanfte Restandardisierung“ \\
Horizontalität & Vertikalisierung & sanfte Devertikalisierung (?) \\
Aggregativität & Integration & sanfte Desintegration (?) \\
8.-15. Jahrhundert & 16.-19. Jahrhundert & 20.-21. Jahrhundert \\
\hline
\end{tabular}

Abb. 1: Phasen der Literalisierung im Deutschen

\section{Folgen für die Grammatik(theorie) I: Wortgruppe und Phrase}

Da die Organisationsprinzipien der Grammatik historisch nicht stabil sind, da sie u.a. in Abhängigkeit von der Literalisierung auf unterschiedlichen Konfigurationen von kognitiven Systemen basieren können, erscheint ein dynamischer Grammatikansatz gerechtfertigt. Ein entsprechendes Forschungsprogramm müsste in der Lage sein, sowohl kontext- als auch symbolgrammatische Modellierungen zu ermöglichen und diese in Abhängigkeit von der jeweiligen Literalisierungsphase aufeinander zu beziehen. Dies bedeutet im Einzelnen:

1. eine kontextgrammatische Modellkomponente für die primäre Oralität;

2. eine symbolgrammatische Modellkomponente für die sekundäre Literalität;

3. die Modellierbarkeit des grammatischen Übergangs zwischen primärer Oralität und sekundärer Literalität, d.h. der primären Literalität;

4. die Modellierbarkeit der Vertikalisierung der kontext- und symbolgrammatischen Modellkomponenten in der sekundären Literalität;

5. die Modellierbarkeit der eventuell kontextgrammatischen Restrukturierung der Symbolgrammatik in der tertiären Literalität.

Um die Tragweite eines solchen Forschungsprogramms abschätzen zu können, möchte ich sie wenigstens an einem zentralen grammatischen Konzept, der Gegenüberstellung von ,Wortgruppe‘ und ,Phrase‘, illustrieren.

16 Der Begriff „sanfte Restandardisierung“, der in etwa dem der „Oraliteralisierung“ (Ágel 2005, S. 104) entspricht, stammt von Koch (2010, S. 198), der von „kleinere(n) Zugeständnisse(n) [...] an Einflüsse aus dem Nähebereich“ (ebd.) spricht. Die Abbildung ist betont schematisch, die Textsortenunterschiede sind mitunter groß. 
In der in den letzten Jahren neu entfachten Diskussion um die Universalgrammatik geht es u.a. um die Frage, ob das Postulat der Universalität der von der Generativen Grammatik favorisierten Variante der Konstituentenstruktur, der $\mathrm{X}$-bar-Theorie, zu halten ist. Eigentlich stellt sich diese Frage nur, wenn man die Struktur des modernen Englisch zum latenten Modell der Universalgrammatik macht. Denn es ist hinlänglich bekannt, dass es eine große Menge von Sprachen mit freier Wortstellung und diskontinuierlicher Wortgruppenbildung gibt, die sich besser dependenziell als konstituentenstrukturell beschreiben lassen (Evans/ Levinson 2009, S. 440 f.). Auch im älteren Deutsch scheint die von Behaghel (1932, S. 240 ff.) sogenannte „Fernstellung“ recht verbreitet gewesen zu sein (siehe unten). Deshalb ist es notwendig, eine typologisch motivierte Unterscheidung zwischen Wortgruppe und Phrase aufzugreifen:

Die Struktur nominaler Ausdrücke ist nicht in allen Sprachen gleich. In einigen Sprachen (z.B. im Lateinischen) sind nominale Ausdrücke locker gefügte Gruppen syntaktisch selbständiger Wörter. In anderen Sprachen (z.B. im Englischen) handelt es sich um hierarchisch strukturierte Phrasen, deren Elemente von unterschiedlicher syntaktischer Selbständigkeit sind. (Himmelmann 1997, S. 1)

In der Geschichte der deutschen Substantivgruppe ist es ein langer Weg von der aggregativen Wortgruppe zur integrativen Phrase. Der entscheidende Umbruch passiert dabei in der Phase der sekundären Literalität, in der sich Phrasen überhaupt erst herausbilden und eine Vertikalisierung von Wortgruppe und Phrase stattfindet. Die einschlägigen Teilprozesse des Syntaxwandels in der Substantivgruppe sind dabei alle mit der Herausbildung und Grammatikalisierung der syntaktischen Nominalklammer im 17./18. Jahrhundert in Zusammenhang zu bringen. ${ }^{17}$

Theoretisch interessanter sind allerdings solche Fälle, die wegen der Übertragung statischer Konzepte auf die Sprachgeschichte nahezu unerkannt bleiben oder statisch interpretiert werden:

(13) [...] als mit Eins ungefehr um 12. Uhr die Ordre kam, unser Regiment, nebst zwey andern (ich glaube Bevern und Kalkstein,) müßten zurückmarschieren. (Bräker III, S. LV, zit. n. Mánássy 2005)

(14) Man zog ihn auf seinen Schlitten nach Haus, wo noch die Mutter samt den Kleinen ihr Wehklagen mit dem unsrigen vereinten. (Bräker III, S. LXIV, zit. n. Mánássy 2005)

17 Insgesamt lassen sich zwölf Teilprozesse nachweisen (Ágel 2000, S. 1858 ff.). 
[...] meine Mutter mit ihren Kindern stehen an der Hausthüre [...] (Haniel IV, S. 19)

Diese Fälle werden in der Regel als sinnkongruente Abweichungen von der Formkongruenz beschrieben. ${ }^{18}$ Aus dynamischer Perspektive geht es hier aber um etwas ganz anderes: Offensichtlich spielt die formale Koordination in diesen Nähetexten noch keine (entscheidende) Rolle. Die Sprachteilhaber kümmern sich nicht um die Wortart der Junktoren, sie kümmern sich also nicht darum, ob es der Konjunktor und ist, der A und B verbindet, oder die komitativen Präpositionen mit, nebst und samt.

Nicht weniger aufschlussreich ist (16), bei dem die Substantivgruppe auf eine leere Menge referiert, aber das Finitum wegen der im Präpositionalattribut ausgedrückten Zehnermenge im Plural steht:

(16) Keins von allen seinen zehn Kinder wollten ihm recht ans Rad stehn (Bräker III, S. LIX)

Ein in gewissem Sinne komplementärer Fall liegt mit (17) vor:

(17) Der Ländertausch im Jahr 1803 und die Bekanntschaft inbetreff der Spedition und der Zahlungen veranlasste mich auf die Hütten Werke der Fürstin zu reflektieren. (Haniel IV, S. 49)

Hier geht es nicht um eine Binominalbildung. Außerdem steht das Subjekt im Vorfeld, sodass die Wortstellung als typologisch bekannter disgruenzfördernder Faktor auch nicht in Frage kommt. Trotzdem kümmert sich der Schreiber um den Konjunktor und nicht, die Wahl des Numerus dürfte auch hier wie beim komplementären Typus semantisch motiviert sein.

In den Fällen (13) bis (17) geht es um das Fehlen derjenigen Kopfeigenschaften, die Zwicky (1993, S. 297 f.) „category determinant“ und „external representative“ genannt hat. ${ }^{19}$ Bei dem Beleg (18) aus dem 14. Jahrhundert fehlt sogar das zentrale syntaktische Merkmal einer Phrase, nämlich der Kopf selbst:

18 Laut Duden (2007, S. 543) übt „das Attribut keinen Einfluss auf den Numerus aus“, weshalb die im Sprachgebrauch durchaus vorhandenen Beispiele grammatisch nicht korrekt seien.

19 Dasselbe gilt für Wortgruppen mit hybriden Substantiven (wie Mädchen oder Fräulein), jedoch mit femininem Pronominalflexiv, die im Nhd.-Korpus von Breder Birkenes/Chroni/Fleischer (2014) (17. bis 19. Jahrhundert) immerhin mit 2,1\% vertreten sind. Z.B. die ältere Fräulein oder nebst seiner Fräulein (ebd., S. 9). 
[...] daz ez dir get an dein ere oder an deinez nechsten (M. v. Amberg 1238 f., zit. n. Ebert 1993, S. 339)

Ebert (ebd.) spricht hier von der „Ersparung des Kernsubstantivs“. Doch ein Kopf ist „required“ (Zwicky 1993, S. 298), darf überhaupt nicht erspart werden. Wie ist die aggregative Organisation hier zu erklären?

Das Possessivum dein in der Substantivgruppe dein ere ist offensichtlich noch ein Pronomen und hat keine Artikelqualitäten wie im Gegenwartsdeutschen. Daraus folgt, dass es sich nicht in einen bestimmten Artikel (die) als Kopf des zweiten Konjunkts und einen Possessivartikel als Kopf des Genitivattributs splitten lässt, was die Voraussetzung für eine Phrasenstruktur wäre.

Bei dem Beleg (19) geht es schließlich um die pronominale Adjektivflexion nach dem bestimmten Artikel:

(19) Aber ich undt der redlicher Geferdt, welcher mit mihr von Oberehn auß reißet, sahen i[h]n nicht. (Güntzer I, S. 6 f., zit. n. Hennig 2009, S. 34)

Morphologisch ist der Kopf einer Phrase nach Zwicky (1993, S. 298) dasjenige Element, das der Ort der morphosyntaktischen Merkmale ist, die die Phrase als Ganzes trägt („morphosyntactic locus“). Im 17. Jahrhundert war dies tendenziell schon der bestimmte Artikel, aber Belege wie (19) zeigen, dass es noch zwei Optionen gab:
(20a) der
redlicher
Geferdt
(20b) der
redliche
Geferdt

Im Falle von (20a) gibt es noch zwei morphologische Kopforte, hier handelt es sich also noch um die Reste einer Wortgruppe. Im Falle von (20b) dagegen ist nur noch der Artikel der morphologische Kopf. ${ }^{20}$

Die Beispieltypen (13) bis (19) repräsentieren also unterschiedliche Typen von Übergängen von der Wortgruppe zur Phrase. Der Prozess der phrasalen Umparametrisierung der Substantivgruppe ist mit der Herausbildung der schriftlichen Leitvarietät im 18. Jahrhundert im Wesentlichen abgeschlossen. Im Sinne der in Abschnitt 2.3 beschriebenen Konsequenzen der Vertikalisierung wird die Strukturoption ,aggregative Wortgruppe in der Schrift- und der späteren Standardsprache aufgegeben und beschränkt sich seit dieser Zeit auf den Substandard (Nähespre-

20 Das Problem ist besonders virulent im Plural, wo die parallele Flexion vom Typ die vergangne Zeiten (Goethe) im älteren Nhd. ,auffallend häufig“ ist (Dal/Eroms 2014, S. 69). 
chen und Dialekte). ${ }^{21}$ Methodisch bedeutet dies jedoch keineswegs, dass unter Behaghels „Fernstellung“ raum- und zeitunabhängig dasselbe zu verstehen wäre. Vergleichen wir die ahd. und mhd. Belege (21) und (22) mit dem ,optisch` gleich aussehenden nähesprachlichen Beleg (23) aus dem 19. Jahrhundert:

(21) wanda ich in dia stat chom der wunterlichun herebergo (N. III, 137, 2, zit. n. Behaghel 1932, S. 243)

(22) daz si getruwe gesellen werden Kristi in sime lidene (Myst. I, 102, 3, zit. n. Behaghel 1932, S. 243)

(23) Da mach doch Gott geben da die Zeit nun endlich mahl komme des Wiedersehns (Briefwechsel V, S. 117)

Admoni (1990, S. 55 ff.) und Schrodt (2004, S. 19 ff.) unterscheiden im Ahd. zwischen kompakten und zusammengesetzten Substantivgruppen. Beide Autoren räumen dabei ein, dass die Grenze wegen der eventuellen Fernstellung von Bestimmungsgliedern unscharf und schwer zu ziehen sei. Ein Grenzproblem liegt allerdings nur vor, wenn man davon ausgeht, dass es bereits im Ahd. Phrasen gab.

Eine Wortgruppe ist

ein Syntagma, in dem syntaktisch selbständige und gleichrangige Elemente juxtaponiert sind. Die Elemente können eine sequentielle und prosodische Einheit bilden, müssen es aber nicht. Das heißt, die Beziehungen der Elemente einer Gruppe untereinander können rein semantischer und ggf. auch morphologischer Natur sein. (Himmelmann 1997, S. 137)

Demnach besteht das methodische Problem im Falle der Fernstellung darin, festzustellen, ob die Bestimmunglieder syntaktisch und semantisch selbstständig sind oder nicht. In den Fällen (21) bis (23) geht es konkret um die Frage der (relativen) Selbstständigkeit des Genitivs im Ahd./Mhd. bzw. im Nhd.

Im älteren Deutsch (und auch im Gotischen) standen sich der possessive und der prädikative Genitiv sehr nahe (Dal/Eroms 2014, S. 31). Mit anderen Worten, auch der verbale Ausdruck der Zugehörigkeitsrelation durch Kopula + Substantivgruppe $_{\text {gen }}$ war, im Gegensatz zum Nhd., produktiv. Aus der Sicht des Genitivs bedeutet dies eine wesentlich größere syntaktische und semantische Selbstständigkeit, denn dieselben Wortgruppen konnten sowohl als nominale Bestimmungsglieder in Fernstellung wie auch als Prädikativa verwendet werden:

21 Die Analogien zum Grammatikwandel im Bereich der Relativpartikeln so und wo sind nicht zu übersehen (siehe Ágel 2010b). 


\section{(21') dia stat ist der wunterlichun herebergo \\ (22') getruwe gesellen sint Kristi}

Der Genitiv im älteren Deutsch war also relativ domänenunspezifisch, die umfassende strukturelle Entwicklung der ,wachsende(n) Differenzierung der Substantiv- und der Verbgruppe“ (Admoni 2002 [1953], S. 49), zu der auch die zunehmende Trennung der strukturellen Verbalkasus (Nom., Akk. und Dat.) vom strukturellen Nominalkasus (Gen.) gehört, setzt erst im Frnhd. ein. ${ }^{22}$

Wendet man nun den obigen ,Prädikativtest' auf (23) an, ist das Ergebnis als fragwürdig einzustufen, da der prädikative Genitivgebrauch im 19. Jahrhundert nicht mehr produktiv ist und da die Substantiv- und die Verbgruppe strukturell weitgehend getrennt sind:

\section{(23’) ?die Zeit ist des Wiedersehns}

Die Fernstellung in (23) ist zwar nähesprachlich, aber die Substantivgruppe gen $_{\text {ist }}$ syntaktisch und semantisch nicht mehr selbstständig, sie ist domänenspezifisch. Hier geht es also nicht um eine aggregative Wortgruppe wie in den ahd. und mhd. Belegen, sondern um die diskontinuierliche Realisierung einer integrativen Phrase. ,Optisch' sehen die Belege vergleichbar aus, sie sind es aber nicht, da die jeweiligen Fernstellungen auf unterschiedlichen strukturellen Grundlagen beruhen.

Der Terminus ,Phrase“ ist in der modernen Linguistik zum Label geworden. Dagegen wäre nichts einzuwenden, wenn jedem, der das Label verwendet, klar wäre, dass ,Phrase‘ ohne weitere Begründung nur ein Etikett ohne theoretischen Wert ist. Das ist aber nicht der Fall. Von dieser inflationären Verwendung des Wortes, die in Termini wie ,Projektorphrase“ oder ,Intonationsphrase‘ gipfelt, profitiert auch die X-bar-Theorie, die uns vormachen will, dass alle Sprachen und Varietäten der Welt in Gegenwart und Geschichte dieselbe syntagmatische Grundstruktur haben. Das ist eine empirisch falsche und theoretisch statische Sicht, die nach der typologischen Untersuchung von Evans/Levinson (2009) zu den Mythen der Universalgrammatik gehört.

22 Da uns keine altdeutschen Muttersprachler zur Verfügung stehen, basiert die postulierte Korrektheit von (21') und (22') auf Analogieschlüssen und der Domänenunspezifik des Genitivs im älteren Deutsch. 


\section{Folgen für die Grammatik(theorie) II: Online- und Offlinesyntax}

\subsection{Die historische Dynamik von Online- und Offlinesyntax}

Wenn die Grammatik im Zuge der Literalisierung umparametrisiert wird, muss dies Konsequenzen auch für unser Verständnis von Mündlichkeit haben, d.h. insbesondere für die Modellierung der Grammatik der Gesprochenen Sprache (Hennig 2009; Zeman 2013) und der sogenannten Differenzgrammatik zwischen gesprochener und geschriebener Sprache (Hennig 2006; Schneider 2011). Auch die Gesprochene-Sprache-Forschung muss also aus der Statizität der Grammatiktheorien ihre Lehren ziehen, um zum dynamischen Perspektivwechsel beitragen zu können.

Gehen wir davon aus, dass Sprecher und Schreiber in Abhängigkeit von der zu lösenden kommunikativen Aufgabe auf diejenigen ihnen verfügbaren sprachlichen Ressourcen zurückgreifen,

a) von denen sie sich erhoffen, diese Aufgabe am besten lösen zu können,

b) die ihnen historisch und soziokulturell überhaupt zur Verfügung stehen und

c) die im jeweiligen Medium überhaupt verwendbar sind. Dies gilt sowohl für die schriftliche Realisierung gesprochensprachlicher Strukturen als auch für die mündliche Realisierung geschriebensprachlicher Strukturen.

Man betrachte hierzu die folgende Abbildung 2. Die Abbildung enthält als Grundmenge alle in einer Varietät verfügbaren syntaktischen Strukturen. So eine (idealisierte) Grundmenge meint man, wenn man beispielsweise von der Syntax des Altsächsischen oder der gegenwartsdeutschen Standardsprache spricht.

Eine Teilmenge dieser syntaktischen Grundmenge ist die Offlinesyntax. Sie ist historisch nicht stabil, sondern wandelt sich in Abhängigkeit von der jeweiligen Phase der Literalisierung; sie ist ein historisch sich wandelndes Gefüge von literalen, d.h. in der jeweiligen Phase der Literalisierung die jeweilige Schriftkultur repräsentierenden, syntaktischen Strukturen. ${ }^{23}$ Darunter, dass offlinesyntaktische Strukturen die jeweilige Schriftkultur repräsentieren, verstehe ich, dass sie in geschriebenen Texten regulär vorkommen, ohne indexikalisch auf die Mündlichkeit zu verweisen. Das wichtigste syntaktische Merkmal des Wandels von offlinesyntaktischen Strukturen ist ihre abnehmende Aggregativität/zunehmende Integrativität.

23 Dies wäre das Schriftlichkeitsanalogon von „Mündlichkeit 3“ nach dem Modell von Zeman (2013). 


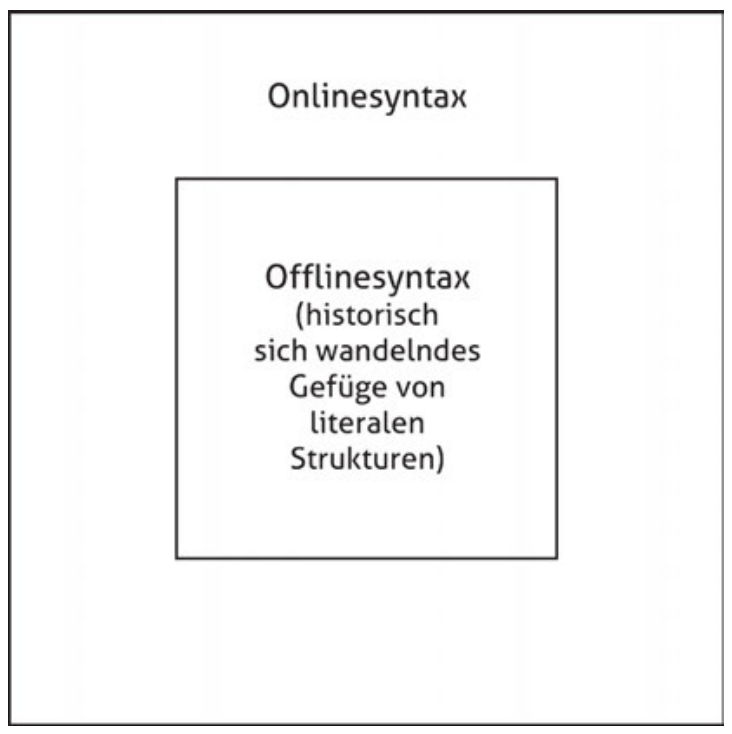

Abb. 2: Die historische Dynamik von Online- und Offlinesyntax ${ }^{24}$

Die Komplementmenge der Offlinesyntax bezogen auf die Grundmenge ist die Onlinesyntax. In der primären Oralität, in der es noch keine Offlinesyntax gibt, ist die Grundmenge mit der Onlinesyntax identisch. Literalisierung bedeutet quantitativ, dass die Offlinesyntax überhaupt erst entsteht und sich auf Kosten der Onlinesyntax ausbreitet.

Aus der Tatsache, dass sich die Offlinesyntax historisch wandelt, folgt, dass sich auch die Komplementmenge der Offlinesyntax, d.h. die Onlinesyntax, historisch wandelt (siehe auch Zeman 2013, S. 397). Mit anderen Worten, in Abhängigkeit von der jeweiligen Phase der Literalisierung wandelt sich auch der Gegenstand der Gesprochenen-Sprache-Forschung. Was unter ,grammatisch mündlich' etwa in Bezug aufs Mhd. zu verstehen ist, muss auf der Basis einer durchweg aggregativen mhd. Offlinesyntax herausgearbeitet werden, während die moderne GesprocheneSprache-Forschung ihre Konzepte vor der Folie einer weitestgehend integrativen standarddeutschen Offlinesyntax entwirft. Beispielsweise stellen (aggregative) Apokoinu-Konstruktionen im Mhd. eine ganz normale offlinesyntaktische Struktur dar, die sicherlich auch online verwendet wurde, während sie in der modernen Standardsprache eine ebenfalls ganz normale onlinesyntaktische Struktur sind.

24 Der Terminus ,Onlinesyntax‘ geht auf Auer (2000) zurück. In einer jüngeren Publikation verwendet er auch den Terminus ,Offlinegrammatik‘ (Auer 2007, S. 95). 
Die onlinesyntaktischen Strukturen operieren also auf den historisch verfügbaren offlinesyntaktischen Strukturen. Die im Rahmen der jeweiligen historischen Grundmenge (,Syntax') vorhandenen primären und sekundären onlinesyntaktischen Strukturen werden also im jeweiligen historischen Online-Offline-Beziehungsgefüge ,ausgehandelt ${ }^{2}{ }^{25}$ Es gibt also keine Apokoinu-Konstruktionen per se, keine Koordinationsellipsen per se, keine Satzrandstrukturen per se usw. Dies mag empirisch und theoretisch trivial sein, aber der methodische Umgang mit Grammatik spricht hier eine ganz andere Sprache. Vielleicht auch deshalb, weil man nur sehr wenigen grammatischen Begriffen ansieht, dass sie historisch gebunden sind. Ein solcher Begriff ist ,Ausklammerung;, dessen Anwendung auf die Zeit vor der Herausbildung der Verbalklammer logischerweise keinen Sinn macht.

Aus diesem dynamischen Online-Offline-Konzept folgt u.a., dass Gesprochene-Sprache-Forscher ihren Gegenstand nicht legitimieren müssen. Die jeweilige historische ,Grenze“ zwischen Online- und Offlinesyntax betrifft Geschriebene- wie Gesprochene-Sprache-Forscher gleichermaßen.

Der Legitimationsdruck, der offenbar auf vielen Gesprochene-Sprache-Forschern lastet, manifestiert sich in Bemühungen, grammatisch korrekte Konstruktionen von sogenannten Performanzfehlern abzugrenzen (siehe etwa Schneider 2011, S. 180 ff.). Grammatikalität ist allerdings ein - in der Regel normatives, da theoriegeleitetes - Konzept, das sich auf die Produkte der Offlinesyntax bezieht (Sampson/Babarczy 2014). Sie lässt sich auf die - typischerweise interaktive onlinesyntaktische Produktion, auf die sich in Gesprächen manifestierende Tätigkeit, nicht übertragen. ${ }^{26}$ Außerdem ist die Bandbreite der onlinesyntaktischen Konstruktionen mitunter wesentlich größer als die der aus offlinesyntaktischen Arbeiten bekannten Strukturen. ${ }^{27}$ Hier lässt sich das Grammatikalitätskonzept, wenn überhaupt, nur auf die online eingesetzten offlinesyntaktischen Strukturen, jedoch nicht auf die genuin onlinesyntaktischen Konstruktionen anwenden. Und Performanzfehler gibt es online (Versprecher) wie offline (Flüchtigkeitsfehler). ${ }^{28}$

25 Das Konzept der primären und sekundären Unterschiede übernehme ich von Hennig (2006, S. 102 ff.), siehe auch Schneider (2011).

26 Nach Coseriu (1988, S. 59) kann „die Tätigkeit des Sprechens, (a) als Tätigkeit, (b) als das der Tätigkeit zugrundeliegende Wissen und (c) als das Produkt der Tätigkeit betrachtet werden [...]“. 27 Instruktiv ist diesbezüglich Auers Analyse der „projizierenden Konstruktionen mit so“ (Auer 2007, S. $109 \mathrm{ff}$.).

28 Kein Geschriebene-Sprache-Forscher kümmert sich um die Frage, grammatisch korrekte Strukturen von Flüchtigkeitsfehlern abzugrenzen. 
Die Gegenstandsfrage für Geschriebene- wie Gesprochene-Sprache-Forscher lautet gleich: Welche syntaktischen Strukturen einer gegebenen historischen Grundmenge in einer gegebenen Phase der Literalisierung sind bereits als Offlinestrukturen grammatikalisiert unabhängig davon, ob sie genetisch aus den jeweiligen Grundbedingungen medialer Mündlichkeit herleitbar sind oder nicht? Frei nach Givon: Today's literacy is yesterday's orality.

Wichtig ist der Bezug auf die gegebene historische Grundmenge. Denn es ist zwar richtig, dass die Gesprochene Sprache die Prozessierung mit der Zeit bevorzugt und deshalb Konstruktionstypen vermeidet, die eine Prozessierung gegen die Zeit erfordern (Auer 2000; 2007). Aber vermeiden kann die gegenwärtige Gesprochene Sprache etwa die Verwendung von sogenannten Rückwärtsellipsen, wie z.B. Klaus arbeitet in und Peter bei Kassel, trivialerweise nur deshalb, weil sich im Deutschen die integrative Struktur ,Rückwärtsellipse‘ überhaupt herausgebildet hat. Umgekehrt kann die gegenwärtige Geschriebene Sprache ApokoinuKonstruktionen trivialerweise nur deshalb vermeiden, weil sich im Deutschen der digital organisierte grammatische Satz (mit diskreten syntaktischen Grenzen) herausgebildet hat (siehe Abschnitt 4.3). ${ }^{29}$

Soweit die Präliminarien. Im Folgenden soll dieses dynamische OnlineOffline-Konzept näher erläutert werden. Im Mittelpunkt steht dabei das Stellungsfeldermodell.

\subsection{Klammern, Stellungsfelder (und die Wortstellung im Ahd.)}

Hinsichtlich der Wortstellung im Ahd. findet man in der ahd. Syntax (Schrodt 2004) recht verwirrende Aussagen:

Im Kapitel „Zweitstellung (Mittelstellung)“ heißt es: „In der normalen, nichtemphatischen Aussage ist die Mittelstellung die Regel“ (ebd., S. 200). Unter Mittelstellung werden dann drei Fälle genannt, wo „,vor dem Vf noch ein weiteres Satzglied (steht)“ (ebd., S. 201). Gemeint ist mit „weiterem Satzglied“, dass vor dem Vf zwei Satzglieder stehen. Diese Fälle werden aber nicht als Verbdrittstellung gerechnet: „Für eine in der Literatur gelegentlich erwähnte grammatische Drittstellung im Aussagesatz gibt es keine sicheren Belege“ (ebd., S. 202, Anm. 1).

In direktem Anschluss, im Kapitel „Späterstellung und Endstellung“ heißt es dann: „Wenngleich die Zweitstellung des finiten Verbs wie im Nhd. schon im Ahd. die Regel war, gibt es doch zahlreiche Belege für Nachzweitstellungen“ (ebd., S. 202).

$29 \mathrm{Zu}$ digitalen vs. analogen Strukturen vgl. Ágel (2003, S. 24 ff.). 
Demnach gibt es also einerseits zwei Satzglieder vor dem Vf, andererseits Nachzweitstellungen des Vf. Diese Stellungen sind aber nicht als Verbdrittstellungen zu werten. Gleich das erste Beispiel für Nachzweitstellung ist ein Beleg aus dem Hildebrandslied:

sunufatarungo iro saro rihtun

,Vater und Sohn ihre Rüstungen richteten' (HL 4)

Schrodt (2004, S. 208 ff.) wendet auch das Stellungsfeldermodell auf das Ahd. an und geht davon aus, dass die nhd. Stellungsfelder und die nhd. Klammer bereits im Ahd. voll da sind: „Grundsätzlich zeigen sich keine großen Unterschiede zwischen ahd. und nhd. Stellungsregularitäten“ (2004, S. 210).

Im Gegensatz zu Schrodt erklärt Hinterhölzl (2010) die ahd. Wortstellung, indem er zeigt, dass die Verbstellung in Abhängigkeit von der Informationsstruktur zu interpretieren ist:
C Hintergrund Kontrastfokus
V
Informationsfokus
(Hinterhölzl 2010, S. 127)

Dieser Befund, wie auch Schlachter (2010) und Schallert (2010), lässt meines Erachtens die Interpretation zu, dass das Verb im Ahd. eine andere, viel autonomere Stellung hatte als später und dass sich die anderen Glieder relativ frei um das Verb herum gruppieren ließen. ${ }^{30}$ Dies bedeutet einen grundlegenden Perspektivwechsel auf die ahd. Wortstellung: Das Verb hat weder eine Erst- noch eine Zweit-, Dritt-, Mittel-, Nachzweit-, Später- oder Letztstellung, sondern steht wie ein Fels in der Brandung grundsätzlich stabil, und es ist das nichtverbale Zeichenmaterial, das sich nach informationsstrukturellen Kriterien um das Verb herum bewegt.

Dieses verbzentrierte Modell, das auf das Stellungsfeldermodell nur schwer abzubilden sein dürfte, lässt sich, ohne die Reihenfolge der nichtverbalen Glieder zu ändern, an Variationen zu dem Beleg (24) wie folgt veranschaulichen:

\footnotetext{
30 „Angesichts der hohen Zahl von Nebensätzen ohne Verbend-Stellung und der Verbdritt-Konstruktionen im deklarativen Hauptsatz wurde eine strukturell verankerte mediale Verbposition vorgeschlagen [...]“ (Schlachter 2010, S. 421). Ahd. stellt „ein reichhaltigeres System dar als spätere Stufen des Deutschen, indem es typische Eigenschaften von VO- und OV-Sprachen miteinander verbindet, also in ontologischer Sicht keinem der beiden Typen angehört“ (Schallert 2010, S. 390). Zu möglichen nichtverbalen Gliedern vor dem Vf in selbstständigen Deklarativsätzen siehe Axel (2002, S. 19 f.), generell zur Verbstellung Dal/Eroms (2014, S. 205 ff.).
} 
(24')

(a) sunufatarungo iro saro

rihtun

(b) sunufatarungo

rihtun iro saro

(c)

rihtun sunufatarungo iro saro

(d) thaz sunufatarungo iro saro

rihtun

(e) thaz sunufatarungo

rihtun iro saro

(f) thaz

rihtun

sunufatarungo iro saro

Unabhängig davon, ob man die verbzentrierte oder die traditionelle verbstellungszentrierte Modellierung der ahd. (bzw. der jüngeren) Wortstellung bevorzugt, theoretisch begründet werden kann ein Modell nur, wenn es für die Formulierung von Regeln notwendig ist (Reis 1980). In diesem Sinne notwendig ist das Stellungsfeldermodell nach Reis (ebd., S. 73 f.), wenn es um die Beschreibung der Abfolge der Pronomina im Gegenwartsdeutschen geht. Allerdings war die Abfolge der Pronomina bis ins frühe Nhd. wesentlich variabler als heute (Behaghel 1932, S. 74 f.), sodass hier keine Notwendigkeit besteht, Abfolgeregeln auf einen (traditionell satztypenneutralen) Mittelfeldbegriff zu beziehen.

Die Notwendigkeit des Regelbezugs ist eine zwar notwendige, aber keine hinreichende Bedingung für die Begründung des Stellungsfeldermodells. Zugrunde liegen muss die Begründung der Klammerstruktur, ohne die es ja keine Stellungsfelder gibt.

An dieser Stelle ist es nicht möglich, die Klammerproblematik ausführlich zu diskutieren, deshalb beschränke ich mich auf drei Punkte (siehe auch Âgel 2000):

1. Obwohl eine Nebensatzklammer unisono - und in der Regel ohne weitere Begründung - angenommen wird, wurden die für die Annahme notwendigen Wortstellungsparallelen zwischen Haupt- und Nebensatz empirisch nie nachgewiesen. Im Gegenteil: Nach aktuellem Forschungsstand haben „sich die zwei Unterarten der Satzklammer nicht ganz gleich entwickelt [...]“ (Ebert 1986, S. 105). ${ }^{31}$ Dies spricht gegen die „methodische Viabilität“ (Ágel 2001, S. 319) eines Klammerbegriffs im weiteren Sinn, der auch die Nebensatzklammer umfasst.

2. Wegen der angeblich komplementären Verteilung von Finitum und Subjunktor in der linken Satzklammer (siehe 3.) ist es zwingend, nur das jeweilige Finitum des Nebensatzprädikats als rechte Satzklammer zu betrachten. Dies

31 Hinterhölzl (2010, S. 136), der die Notker-Daten von Näf (1979) interpretiert, stellt sogar „eine eklatante Diskrepanz in der Herausbildung der Satzklammer zwischen Hauptsätzen und Nebensätzen“ fest: In Hauptsätzen werden Subjekte viermal so oft und Objekte fast doppelt so oft ausgeklammert wie in Nebensätzen. 
führt dazu, dass der Infinitivkomplex in einer Reihe von Verbalkomplexen obligatorisch ,ausgeklammert" ist, z.B. ... [[dass] sie [hat]] arbeiten können. Somit wird theoretisch eine Art Verdoppelung des Ausklammerungsbegriffs erzwungen, denn neben der ,externen', außerhalb der rechten Satzklammer stattfindenden, Ausklammerung gibt es auch eine Art ,interner Ausklammerung' im Verbalkomplex selbst. ${ }^{32}$

3. Auch wenn die linke Satzklammer nur eine „Positionskategorie“ (Wöllstein 2010, S. 26) sein soll, ist die komplementäre Verteilung von Finitum und Subjunktor in der linken Satzklammer höchst fragwürdig. Komplementarität lässt sich nämlich allenfalls zwischen dem V1- und dem Vletzt-Satztyp, (26a) vs. (26b), nicht jedoch zwischen V2 und Vletzt, (26c) vs. (26b), herstellen:

$\begin{array}{llllll}\text { (26a) } & {[[\text { Hat }]} & \text { man } & \text { dich } & \text { [erwischt]?] } & \\ \text { (26b) } & {[[\text { dass }]} & \text { man } & \text { dich } & \text { erwischt } & \text { [hat] } \\ \text { (26c) } & \text { Man } & \text { [[hat }] & \text { dich } & \text { [erwischt].] } & \end{array}$

Wöllstein (ebd., S. 26) führt die Komplementarität tatsächlich anhand der Gegenüberstellung der Beispiele (26a) und (26b), also von V1 und Vletzt, ein, aber in der anschließenden Tabelle, die die komplementäre Verteilung von Finitum und Subjunktor veranschaulichen soll (ebd., S. 27), werden nicht mehr V1 und Vletzt, sondern V2 und Vletzt gegenübergestellt.

Unklar bleibt bei dieser Art Komplementaritätspostulat nicht nur, worauf die basale topologische Gemeinsamkeit von V1 und Vletzt (syntaktisch und/oder semantisch und/oder pragmatisch) beruht, sondern auch, wie sich angesichts der Miteinbeziehung von V2 in das Komplementaritätspostulat die Exklusivität des V2-Vorfelds begründen lässt.

Geht man dagegen davon aus, dass sich die Wortstellungsregularitäten im Nebensatz ohne Klammer erfassen lassen, bleiben nur die grammatisch einheitlichen, da prädikatsbildenden, Verbklammern der Satztypen (26a) und (26c) übrig.

Sind aber alle Prädikate, die nichtverbale Glieder ,umklammern', gleich als Verbklammern zu werten?

$\mathrm{Zu}$ unterscheiden ist mit Eichinger (1995, S. 304) zwischen Distanzstellung und Klammer. Unter Klammern versteht er Konstruktionen „mit einem spezifisch strukturierten ,Inhalt““ zwischen den beiden Klammerteilen. Mit anderen Wor-

32 Zum Bech'schen Unterfeld siehe auch Abschnitt 4.3. 
ten, Klammerteile umschließen kein ungeordnetes Aggregat von Elementen, sondern ein Mittelfeld, dessen Kriterium die spezifische Anordnung seiner Elemente ist. Unter Distanzstellung versteht er hingegen lediglich die Fernstellung von grammatisch zusammengehörigen Konstituenten voneinander. Die Grammatikalisierung der Distanzstellung findet im Frnhd. und im frühen Nhd. statt, die nach Eichinger erst zu Beginn des 18. Jahrhunderts in die Herausbildung der Klammer mündet.

Eichinger (ebd., S. 321) erklärt den Wechsel von der Distanzstellung zur Klammer „mit den grundsätzlich gewandelten kommunikativen Ansprüchen einer veränderten Öffentlichkeit“. Was heißt das?

Im 17. Jahrhundert, wo das Prinzip der Distanzstellung bereits weitestgehend durchgeführt ist, herrsche immer noch ein eher anreihender Strukturtyp vor (ebd., S. 313 ff.). Anreihung ist ein Strukturprinzip, das ursprünglich für die konzeptionelle Mündlichkeit charakteristisch war. Ihre Übertragung in die konzeptionelle Schriftlichkeit in der Kanzleisprache führt zur Interferenz mit der immer mehr überdehnten und im 17. Jahrhundert schon grammatikalisierten Distanzstellung (ebd., S. 315 ff.); sie führt nahezu unvermeidlich ,zu ziemlich schwerverständlichen Konstruktionen“ (ebd., S. 315).

Diese Rezeptionsschwierigkeiten stellen solange kein Problem dar, als „das in den entsprechenden Schriften vermittelte Wissen von öffentlichen Dingen Herrschafts- und Spezialistenwissen ist“ (ebd., S. 317). Unter den „grundsätzlich gewandelten kommunikativen Ansprüchen einer veränderten Öffentlichkeit“ werde jedoch das markierte Strukturprinzip der Kanzleisprache durch einen neuen Schub konzeptioneller Mündlichkeit repariert.

In diesem Kontext spielt nach Eichinger Reichmanns vierte - die sprachreflexive - Vertikalisierungsdimension die entscheidende Rolle:

Die Zeit der Vertikalisierung (16.-18. Jahrhundert) fällt mit der der nationalkulturellen und patriotischen Instrumentalisierung von Sprache, einem neuen sprachreflexiven Denken, zusammen. Der Leitvarietät werden dabei besondere Gütequalitäten zugeschrieben, die anderen Varietäten nicht zukommen. Nach der rationalistischen Auffassung von einem möglichst ungebrochenen Entsprechungsverhältnis zwischen Sachen/Sachverhalten, Gedanken und Sprachzeichen setzt das deutliche und eindeutige Sprechen und Schreiben aufgeklärter, gebildeter Bürger u.a. deutliche syntaktische Regeln voraus (Reichmann 1995, S. 188). Eine in diesem Sinne deutliche Regel im ausgehenden 17. Jahrhundert ist nach Eichinger die Distanzstellung der analytischen Verbformen. Deutlich musste nur noch das werdende Mittelfeld strukturiert werden, um die Realisierung des idealen rationalistischen Entsprechungsverhältnisses syntaktisch zu ermöglichen. Dies ist dann im 18. Jahrhundert erfolgt (mehr dazu in Ágel 2000). 


\subsection{Entzeitlichung, Linearisierung, Integration}

Im Sinne des in Abschnitt 4.2 Gesagten ist davon auszugehen, dass es vor dem 18. Jahrhundert keine Klammerstrukturen und Stellungsfelder im heutigen Sinne gab und dass deren Grammatikalisierung eng mit der sekundären Literalität verbunden ist, da sie sich nur vor der Folie der (natürlich idealisierten) Opposition von konzeptioneller Mündlichkeit und konzeptioneller Schriftlichkeit modellieren lässt.

Was sind nun die Konsequenzen für ein dynamisches Online-OfflineKonzept?

Richtungsweisend ist hier die von Günthner und Hopper getroffene Unterscheidung zwischen der Zeitlichkeit der gesprochenen und der entzeitlichten Linearität der geschriebenen Sprache:

Die Linearität des Zeichens, von dem de Saussure - trotz seiner Betonung der Zeitlichkeit gesprochener Sprache - ausging, ist ein entzeitlichtes Konzept, das auf der Metapher des geschriebenen Textes als fertiges Produkt basiert, wobei unsere horizontale Ausrichtung beim Lesen ikonisch als Progression in der Zeit interpretiert wird. (Günthner/Hopper 2010, S. 3)

Die Stellungsfelder, d.h. Vor-, Mittel- und Nachfeld, stellen ein Produkt der entzeitlichten Linearität der konzeptionellen Schriftlichkeit dar, sie gehören also zur Offlinesyntax. Die sogenannten Satzrandstrukturen, die sich nicht als Felder grammatikalisiert haben, stellen dagegen natürliche Ressourcen der Onlinesyntax dar, sie sind Produkte der Zeitlichkeit der Onlinesyntax, die an den Rändern der offlinesyntaktischen Stellungsfelder operieren. Sätze als Offlinestrukturen der sekundären Literalität sind also online „prinzipiell verlängerbar“ (Selting 1995, S. 73). ${ }^{33}$ Ich schlage für die Bezeichnung der gegenwartsdeutschen Onlinestrukturen am Rande der Offlinesätze den Terminus (linkes und rechtes) Satzrandglied vor, um diese Strukturen auch terminologisch von den Offlinesatzgliedern zu unterscheiden.

Eine mögliche Einbindung der Raumstruktur der Stellungsfelder in die Zeitstruktur der Onlinesyntax soll an der Kombination eines Timm-Belegs mit einigen typischen linken Satzrandgliedern veranschaulicht werden:

(27) Ich komme aus dem Garten in die Küche, wo die Erwachsenen stehen, meine Mutter, mein Vater, meine Schwester. (Timm Bruder, S. 7)

$33 \mathrm{Zu}$ Satz als prototypische Offlinestruktur siehe auch Maas (2010). 


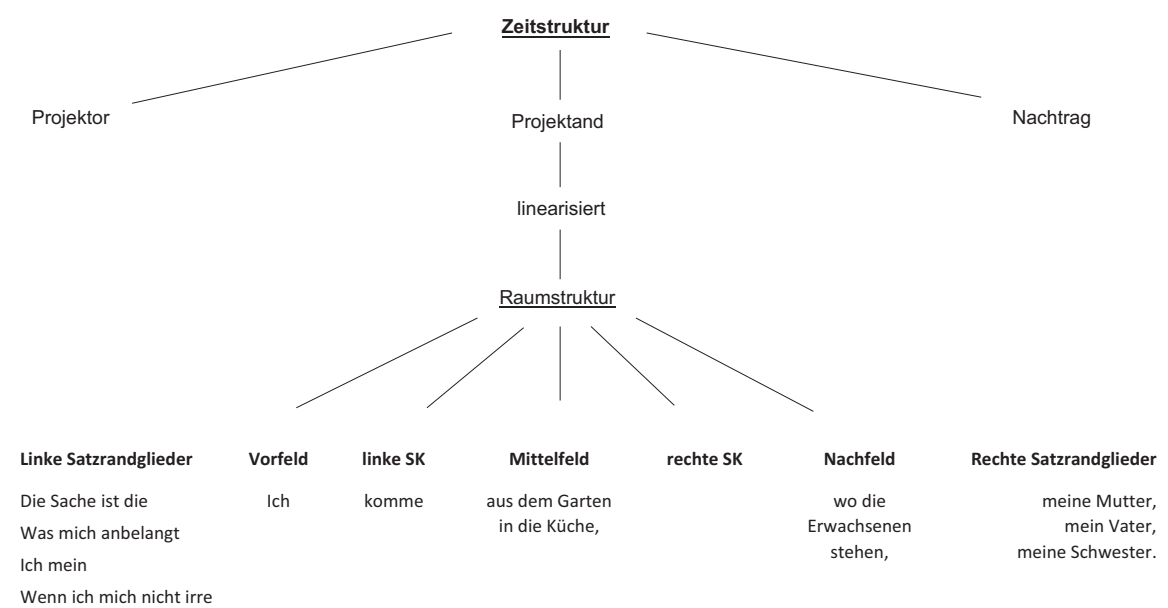

Abb. 3: Offline-Stellungsfelder als Online-Ressource

Eine potenzielle Online-Grundstruktur besteht aus drei (zeitlich) nacheinander zu realisierenden Teilen: Aus den beiden Teilen einer sogenannten Projektorkonstruktion (Günthner 2008), die ich Projektor und Projektand nenne, und dem Nachtrag, unter dem die zeitliche Verlängerung einer Projektorkonstruktion zu verstehen ist.

Mögliche Projektoren der in Abbildung 3 modellierten Onlinestruktur sind die angeführten linken Satzrandglieder, deren Projektand der grammatische Satz, d.h. die durch die Stellungsfelder abgegrenzte Offlinestruktur, ist. Nachgetragen wurden hier die rechten Satzrandglieder meine Mutter, mein Vater, meine Schwester. Diese stellen die ikonische Umfunktionierung einer genuinen Online-Ressource als Teil derjenigen grammatischen Strukturen bei Timm dar, die Erinnerungen simulieren (George 2014). Mit anderen Worten, in (27) wird eine genuine Online-Struktur offline genutzt.

Der grammatische Satz, der typischerweise Projektand einer Online-Struktur ist, ist intern integrativ organisiert: Die Klammer und die Stellungsfelder bilden einen Systemraum, in dem die Linearisierungsregeln aufeinander abgestimmt sind.

Die Satzgrenzen, d.h. die Grenzen zwischen Projektor und Projektand bzw. Projektand und Nachtrag, sind dagegen typischerweise aggregativ, es sind die Nahtstellen der Einbindung der prototypischen Raumstruktur ,Satz' in die dreigliedrige Zeitstruktur.

Gibt es aber Evidenzen für

a) den genuinen Offline-Charakter und

b) die späte historische Herausbildung der Stellungsfelder? 
Indirekte Evidenz für den genuinen Offline-Charakter liefern u.a. Adjazenzstrukturen, z.B.
A: Woher kommst du?
B: Aus Mannheim ( $\neq$ Aus Mannheim komme ich)

Zeitlichkeit ist das eine zentrale Merkmal gesprochener Sprache, das andere ist Dialogizität. In der Tat funktionieren Frage-Antwort-Strukturen wie (28) nicht im Sinne des Offline-Stellungsfeldermodells. Denn Bs Antwort wird nicht so verstanden, dass das Antwort-Direktivum das Frage-Direktivum paradigmatisch ersetzt. Mit anderen Worten, obwohl Woher das Vorfeld besetzt, wird aus Mannheim nicht als Vorfeldbesetzung interpretiert.

Was b) anbelangt, soll kurz auf 1) die Herausbildung linker Satzrandglieder und 2) das verbale Schlussfeld im Nebensatzprädikat eingegangen werden.

\section{Ad 1):}

Linksversetzungen stellen nach Hennig (2009, S. 36) universelle Nähemerkmale dar, da ihre Verwendung „offenbar keinen besonderen historischen Bedingungen unterliegt“. Entsprechend kommen sie bzw. generell Linksherausstellungen auch „in recht unterschiedlichen Sprachen“ vor (Lötscher 1995, S. 35 f.). Im Deutschen sind Prolepsen bereits aus ahd. Zeit belegt (Näf 1979, S. 128 ff.), wo sie bei Notker „eine gewisse Nähe zur gesprochenen Sprache“ zeigen (Näf 1979, S. 131). Im Mhd. waren Linksherausstellungen selten (Dal/Eroms 2014, S. 213). Im Frnhd., in der Zeit der Grammatikalisierung der Distanzstellung, nimmt der Gebrauch bis Anfang des 16. Jahrhunderts kontinuierlich ab (Lötscher 1995, S. 42), was Lötscher (ebd., S. 55) mit der Herausbildung der konzeptionellen Schriftlichkeit erklärt. Im Nhd. sind sie nur noch aus Nähetexten bekannt (siehe Elspaß 2005 und 2010).

Wenn nun die Stellungsfelder im heutigen Sinne erst seit dem 18. Jahrhundert zur Verfügung stehen, bedeutet dies, dass der gegenwartsdeutsche grammatische Satz, dessen Definiens (u.a.) die Stellungsfelder sind, eine relativ junge Struktur ist. Entsprechend jung sind die Satzrandglieder, die sich als (spezifisch deutsche) Grammatikalisierungen von Linksherausstellungen beschreiben lassen. Linksherausstellungen sind demnach Onlineprojektoren, die sich auf die On- oder OfflineVorgängerprojektanden des heutigen Satzes beziehen. Linke Satzrandglieder sind dagegen Online-Projektoren (auch) des ,modernen` Offlineprojektanden ,Satz ‘ ${ }^{34}$ Wie man an der kurzen historischen Skizze sieht, waren Linksherausstellungen unproblematisch in der Phase der primären Literalität, in der es noch keine Sätze

34 Natürlich projizieren linke Satzrandglieder nicht nur Sätze. 
im heutigen Sinne gab. In der sekundären und tertiären Literalität beziehen sich diese Zeitstrukturen allerdings auf eine Raumstruktur, was kontraikonisch ist.

Soweit die Theorie. Und die Praxis? Gibt es diagnostische Möglichkeiten, zwischen (früheren) Linksherausstellungen und (heutigen) linken Satzrandstrukturen zu unterscheiden?

Nach Behaghel (1932, S. 14) steht im ältesten Ahd. „das Verbum nicht in Zweitstellung, wenn der Satz außer dem einleitenden Wort noch unbetonte Wörtchen enthält", vgl.

dhiu chiborgonun hort dhir gibu (Isid(or) 6, 4, ebd.)

Dass Prolepsen vergleichbar strukturiert waren, ist nicht zu übersehen, z.B.

(30) Intentio unde depulsio. / diu machônt / ten statum. (Notker, AK 79.21, zit. n. Näf 1979, S. 129)

Würde man Intentio unde depulsio als Vorvorfeldbesetzung interpretieren, müsste man entsprechend auch das Akkusativobjekt dhiu chiborgonun hort als Vorvorfeldbesetzung auffassen oder eben in beiden Fällen davon ausgehen, dass das jeweilige Vorfeld doppelt besetzt ist.

Dass zwei Glieder vor dem Finitum lange Zeit einfach juxtaponiert waren und kein hierarchisches Verhältnis eingingen, davon zeugt auch die Geschichte der sogenannten linksperipheren Adverbial(neben)sätze (= AS). Nach Axel (2002, S. 12)

waren AS bis ins Spätmhd. hinein offenbar nicht vorfeldfähig, denn die V2-Stellung ist nahezu überhaupt nicht belegt [...]. Dies spricht dafür, die weitverbreitete resumptive Struktur [...] als Realisierungsvariante von V3 [= Verbdrittstellung, V.Á.] einzustufen.

Dafür, dass sie nicht vorfeldfähig waren, gibt es eine noch einfachere Erklärung: Es gab kein Vorfeld.

In dem nhd. Nähekorpus von Kappel (zuletzt 2014) finden sich auch nichtresumptive V3-Belege:

(31) Weil wir den vor Augen stehende[n] reichen-Seegen den Som[m]er über angeschaut, mann vermeinet, Es sollte wohlfailer werden. (Schuhmacher Chronik II, S. 343 f., zit. n. Kappel 2014, S. 210)

Integration der AS setzt die Herausbildung des Vorfeldes voraus. Nähebelege wie (31) (aus der ersten Hälfte des 18. Jahrhunderts) deuten darauf hin, dass die aggregative grammatische Struktur ohne Einbettungsmöglichkeit der AS, d.h. ohne Vorfeld, zunehmend von der integrativen verdrängt und überlagert wurde. 
Ad 2):

Die Herausbildung des verbalen Schlussfelds stellt ein perfektes Beispiel für die Grammatikalisierung eines Aggregatraumes dar. ${ }^{35}$

$$
\begin{aligned}
& \text {... dass er [getroffen } \left.\text { worden }_{3} \text { sein }_{2} \text { soll }_{1}\right]_{\text {Schlussfeld }} \\
& \text {... dass sie }\left[\left[\text { soll }_{1} \text { haben }\right]_{\text {Oberfeld }}\left[\text { kommen }_{4} \text { können }_{3}\right]_{\text {Unterfeld }}\right]_{\text {Schlussfeld }}
\end{aligned}
$$

Nach den gegenwartsdeutschen Serialisierungsnormen ist das verbale Schlussfeld entweder durchgehend linksdependent, oder es zerfällt in ein rechtsdependentes Oberfeld und ein linksdependentes Unterfeld.

Dies war im älteren Deutsch und zum Teil auch noch im Frnhd. anders. Nach Askedal (1998) dominiert in den dreigliedrigen Verbalkomplexen der Berthold von Regensburg (13. Jahrhundert) zugeschriebenen deutschen Predigten eindeutig die Zwischenstellung des Finitums (63,2\%), z.B.

$$
\text { ob dû der liute bist die bekêrt }{ }_{3} \operatorname{suln}_{1} \text { werden }_{2} \text { (zit. n. Askedal 1998, S. 243) }
$$

Askedal stellt zu Recht fest, dass zu Bertholds Zeiten „eine Kohärenz-InkohärenzOpposition, wie sie in der modernen deutschen Standardsprache vorliegt, sich noch nicht herausgebildet hat“ (ebd., S. 254).

In einer auf breiter Materialgrundlage durchgeführten Studie, die aber erst mit 1450 ansetzt, kommt Härd (1981, S. 55) zu dem Ergebnis, dass die Zwischenstellung „als ein Charakteristikum des deutschen Prosastils im 15. und 16. Jahrhundert bezeichnet werden (darf)“. Doch seit etwa 1525 zeige dieser Strukturtyp eine markant sinkende Frequenz (ebd.). Im Laufe des 17. Jahrhunderts wird sie dann ,aus der auf überregionale Geltung Anspruch erhebenden Schriftsprache beinahe vollständig verdrängt und auf Restgebiete beschränkt“ (ebd., S. 89).

Den Untersuchungsergebnissen von Askedal und Härd ist zu entnehmen, dass das verbale Schlussfeld ein typisches Offlineprodukt der Phase der sekundären Literalität ist. Voraussetzung für seine Herausbildung ist ein visueller Raum der entzeitlichten Linearität. Die Zwischenstellung wird im Zuge der Vertikalisierung an die Nähetexte weitergereicht und ist in der Onlinesyntax - und natürlich auch in den Dialekten - bis heute vorhanden.

Die heutige Zwischenstellung ist aber strukturell nicht zu vergleichen mit der Zwischenstellung zu Bertholds Zeiten. Die damalige Zwischenstellung ist Teil einer durchgehend aggregativen verbalen Schlussstruktur. Die heutige Zwischen-

35 Zum Schlussfeld siehe Bech (1983 [1955-1957], S. 62), zur Serialisierung der Nebensatzprädikate Ágel (2001). 
stellung in der Onlinesyntax ist dagegen analog zum Verhältnis der Offline-Stellungsfelder zu den Online-Satzrandgliedern zu interpretieren: Die Zwischenstellung stellt eine natürliche Ressource der Onlinesyntax dar, das Offline-Schlussfeld interaktiv aufzubrechen.

\section{Fazit im Telegrammstil}

1. Literalisierung ist eine kulturelle Universalie, die kognitiv verankert ist.

2. Es sind unterschiedliche Phasen der Literalisierung zu unterscheiden.

3. Die Interpretation von grammatischen Strukturen ist nur vor der Folie der jeweiligen Phase der Literalisierung möglich.

4. Literalisierung im Allgemeinen und die Phasen der Literalisierung im Besonderen führen zur Umparametrisierung und Vertikalisierung der grammatischen Struktur: Aggregative Strukturen werden von integrativen verdrängt und/oder überlagert; zeitliche Strukturen werden von entzeitlichten (linearisierten) verdrängt und/oder überlagert.

5. Prestigeträger, an denen sich grammatische Laien wie Grammatiktheoretiker orientieren, sind die integrativen und entzeitlichten Strukturen.

6. Historisch ist von einem dynamischen Verhältnis von Online- und Offlinesyntax auszugehen. Was zu einer bestimmten Zeit und in einer bestimmten Varietät als Onlinestruktur zu interpretieren ist, hängt von dem jeweiligen historischen Verhältnis von Online- und Offlinestrukturen ab. ${ }^{36}$

\section{Quellen und Literatur}

\section{Quellen}

Bauernleben I = Eckhardt, Wilhelm A./Klingelhöfer, Helmut (Hg.) (1998): Bauernleben im Zeitalter des Dreißigjährigen Krieges. Die Stausebacher Chronik des Caspar Preis 16361667. Mit einer Einführung von Gerhard Menk. (= Beiträge zur Hessischen Geschichte 13). Marburg.

Bräker III = Bräker, Ulrich (1985 [1789]): Lebensgeschichte und natürliche Abenteuer des Armen Mannes im Tockenburg. Berlin.

36 Für wichtige Hinweise und Kommentare danke ich Hans-Werner Eroms, Jürgen Erich Schmidt und Johannes Schwitalla. 
Briefwechsel V = Schikorsky, Isa (Hg.) (1999): „Wenn doch dies Elend ein Ende hätte“: ein Briefwechsel aus dem Deutsch-Französischen Krieg 1870/71. (= Selbstzeugnisse der Neuzeit 7). Köln u.a.

Güntzer I = Brändle, Sebastian (Hg.) (2002): Güntzer, Augustin (1657): Kleines Biechlin von meinem gantzen Leben. Die Autobiographie eines Elsässer Kannengießers aus dem 17. Jahrhundert. (= Selbstzeugnisse der Neuzeit 8). Köln u.a.

Haniel IV = Herzog, Bodo/Mattheier, Klaus J. (Hg.) (1979): Haniel, Franz 1779-1868.

Materialien, Dokumente und Untersuchungen zu Leben und Werk des Industriepioniers Franz Haniel. (= Veröffentlichungen des Instituts für Geschichtliche Landeskunde der Rheinlande der Universität Bonn). Bonn.

Timm Bruder = Timm, Uwe (2006): Am Beispiel meines Bruders. München.

\section{Literatur}

Aaron, P.G./Joshi, R. Malatesha (2006): Written language is as natural as spoken language: a biolinguistic perspective. In: Reading Psychology 27, S. 263-311.

Admoni, Wladimir G. (1990): Historische Syntax des Deutschen. Tübingen.

Admoni, Wladimir G. (2002 [1953]): Zu einigen Gesetzmäßigkeiten der Entwicklung des syntaktischen Baus der Sprache. In: Admoni, Wladimir G.: Sprachtheorie und deutsche Grammatik. Aufsätze aus den Jahren 1949-1975. Hrsg. v. Pavlov, Vladimir/Reichmann, Oskar. Tübingen, S. 35-61. [Sowjetische Originalausg. 1953 in (übersetzt): Beiträge und Mitteilungen des Instituts für Sprachwissenschaft der Akademie der Wissenschaften der UdSSR, S. 27-52.]

Ágel, Vilmos (1999): Grammatik und Kulturgeschichte. Die raison graphique am Beispiel der Epistemik. In: Gardt, Andreas/Haß-Zumkehr, Ulrike/Roelcke, Thorsten (Hg.): Sprachgeschichte als Kulturgeschichte. (= Studia Linguistica Germanica 54). Berlin/New York, S. 171-223.

Ágel, Vilmos (2000): Syntax des Neuhochdeutschen bis zur Mitte des 20. Jahrhunderts. In: Besch, Werner et al. (Hg.): Sprachgeschichte. Ein Handbuch zur Geschichte der deutschen Sprache und ihrer Erforschung. 2. Halbbd. 2. Aufl. (= Handbücher zur Sprach- und Kommunikationswissenschaft 2). Berlin/New York, S. 1855-1903.

Ágel, Vilmos (2001): Gegenwartsgrammatik und Sprachgeschichte. Methodologische Überlegungen am Beispiel der Serialisierung im Verbalkomplex. In: Zeitschrift für Germanistische Linguistik 29, S. 319-331.

Ágel, Vilmos (2003): Prinzipien der Grammatik. In: Lobenstein-Reichmann, Anja/Reichmann, Oskar (Hg.): Neue historische Grammatiken. Zum Stand der Grammatikschreibung historischer Sprachstufen des Deutschen und anderer Sprachen. (= Reihe Germanistische Linguistik 243). Tübingen, S. 1-46.

Ágel, Vilmos (2005): Wort-Arten aus Nähe und Distanz. In: Knobloch, Clemens/Schaeder, Burkhard (Hg.): Wortarten und Grammatikalisierung. Perspektiven in System und Erwerb. (= Linguistik - Impulse \& Tendenzen 12). Berlin/New York, S. 95-129.

Ágel, Vilmos (2007): Was ist „grammatische Aufklärung“ in einer Schriftkultur? Die Parameter „Aggregation“ und „Integration“. In: Feilke, Helmuth/Knobloch, Clemens/Völzing, Paul-Ludwig (Hg.): Was heißt linguistische Aufklärung? Sprachauffassungen zwischen Systemvertrauen und Benutzerfürsorge. (= Wissenschaftskommunikation 1). Heidelberg, S. 39-57. 
Ágel, Vilmos (2010a): Explizite Junktion. Theorie und Operationalisierung. In: Ziegler (Hg.) (2010b), S. 897-936.

Ágel, Vilmos (2010b): +/-Wandel. Am Beispiel der Relativpartikeln so und wo. In: Bittner, Dagmar/Gaeta, Livio (Hg.): Kodierungstechniken im Wandel. Das Zusammenspiel von Analytik und Synthese im Gegenwartsdeutschen. (= Linguistik - Impulse \& Tendenzen 34). Berlin/New York, S. 199-222.

Ágel, Vilmos/Diegelmann, Carmen (2010): Theorie und Praxis der expliziten Junktion. In: Ágel, Vilmos/Hennig, Mathilde (Hg.): Nähe und Distanz im Kontext variationslinguistischer Forschung. (= Linguistik - Impulse \& Tendenzen 35). Berlin, S. 347-396.

Ágel, Vilmos/Hennig, Mathilde (Hg.) (2006): Grammatik aus Nähe und Distanz. Theorie und Praxis am Beispiel von Nähetexten 1650-2000. Tübingen.

Askedal, John Ole (1998): Zur Syntax infiniter Verbalformen in den Berthold von Regensburg zugeschriebenen deutschen Predigten. Vorstufe der topologischen Kohärenz-InkohärenzOpposition? In: Askedal, John Ole (Hg.): Historische germanische und deutsche Syntax. Frankfurt a.M. u.a., S. 231-259.

Auer, Peter (2000): Online Syntax - oder: was es bedeuten könnte, die Zeitlichkeit der mündlichen Sprache ernst zu nehmen. In: Sprache und Literatur 85, S. 43-56.

Auer, Peter (2007): Syntax als Prozess. In: Hausendorf, Heiko (Hg.): Gespräch als Prozess. Linguistische Aspekte der Zeitlichkeit verbaler Interaktion. (= Studien zur Deutschen Sprache 37). Tübingen, S. 95-124.

Axel, Katrin (2002): Zur diachronen Entwicklung der syntaktischen Integration linksperipherer Adverbialsätze im Deutschen: Ein Beispiel für syntaktischen Wandel? In: Beiträge zur Geschichte der deutschen Sprache und Literatur 124, S. 1-43.

Bech, Gunnar (1983 [1955-1957]): Studien über das deutsche Verbum infinitum. 2., unveränd. Aufl. (= Linguistische Arbeiten 139). Tübingen. [1. Aufl. Kopenhagen 1955-1957.]

Behaghel, Otto (1932): Deutsche Syntax. Eine geschichtliche Darstellung. Bd. 4. (= Germanistische Bibliothek, Abt. Sammlung germanischer Elementar- und Handbücher, Grammatiken 10). Tübingen.

Breder Birkenes, Magnus/Chroni, Kleopatra/Fleischer, Jürg (2014): Genus- und Sexuskongruenz im Neuhochdeutschen: Ergebnisse einer Korpusuntersuchung zur narrativen Prosa des 17. bis 19. Jahrhunderts. In: Deutsche Sprache 42, S. 1-24.

Coseriu, Eugenio (1974): Synchronie, Diachronie und Geschichte. Das Problem des Sprachwandels. (= Internationale Bibliothek für Allgemeine Linguistik 3). München.

Coseriu, Eugenio (1988): Sprachkompetenz. Grundzüge der Theorie des Sprechens. (= Uni-Taschenbücher 1481). Tübingen.

Dal, Ingerid/Eroms, Hans-Werner (2014): Kurze deutsche Syntax auf historischer Grundlage. 4. Aufl. Neu bearbeitet von Hans-Werner Eroms. (= Sammlung kurzer Grammatiken germanischer Dialekte B/7). Berlin/Boston.

Duden (2007): Der Duden. Bd. 9: Richtiges und gutes Deutsch. Wörterbuch der sprachlichen Zweifelsfälle. 6., vollst. überarb. Aufl. Mannheim u.a.

Ebert, Robert Peter (1986): Historische Syntax des Deutschen. Bd. 2: 1300-1750. (= Germanistische Lehrbuchsammlung 6). Bern u.a.

Ebert, Robert Peter (1993): Syntax. In: Reichmann, Oskar/Wegera, Klaus-Peter (Hg.): Frühneuhochdeutsche Grammatik. (= Sammlung kurzer Grammatiken germanischer Dialekte A/12). Tübingen, S. 313-484.

Eichinger, Ludwig M. (1995): Syntaktischer Wandel und Verständlichkeit. Zur Serialisierung von Sätzen und Nominalgruppen im frühen Neuhochdeutschen. In: Kretzenbacher, Heinz L./ Weinrich, Harald (Hg.): Linguistik der Wissenschaftssprache. Berlin/New York, S. 301-324. 
Eisenberg, Peter (1995): Grammatik der geschriebenen Sprache als Symbolgrammatik. Ein Versuch an ausgewählten Themen der Morphologie. In: Ágel, Vilmos/Brdar-Szabó, Rita (Hg.): Grammatik und deutsche Grammatiken. (= Linguistische Arbeiten 330). Tübingen, S. 23-38.

Eisenberg, Peter (2007): Sollen Grammatiken die gesprochene Sprache beschreiben? Sprachmodalität und Sprachstandard. In: Ágel, Vilmos/Hennig, Mathilde (Hg.): Zugänge zur Grammatik der gesprochenen Sprache. (= Reihe Germanistische Linguistik 269). Tübingen, S. 275-295.

Elspaß, Stephan (2005): Sprachgeschichte von unten. Untersuchungen zum geschriebenen Alltagsdeutsch im 19. Jahrhundert. (= Reihe Germanistische Linguistik 263). Tübingen.

Elspaß, Stephan (2010): Klammerstrukturen in nähesprachlichen Texten des 19. und frühen 20. Jahrhunderts. Ein Plädoyer für die Verknüpfung von historischer und Gegenwartsgrammatik. In: Ziegler (Hg.) (2010b), S. 1011-1026.

Enfield, Nick J. (2002): Ethnosyntax: Introduction. In: Enfield, Nick J. (Hg.): Ethnosyntax: Explorations in culture and grammar. Oxford, S. 1-30.

Evans, Nicholas/Levinson, Stephen C. (2009): The myth of language universals: Language diversity and its importance for cognitive science. In: Behavioral and Brain Sciences 32, S. 429-492.

Everett, Daniel L. (2005): Cultural constraints on grammar and cognition in Pirahã. Another look at the design features of human language. In: Current Anthropology 46, S. 621-646.

Everett, Daniel L. (2010): Das glücklichste Volk. Sieben Jahre bei den Pirahã-Indianern am Amazonas. München. [Engl. Originalausg. London 2008: Don't sleep, there are snakes: Life and language in the Amazonian jungle.]

Everett, Daniel L. (2013): Die größte Erfindung der Menschheit. Was mich meine Jahre am Amazonas über das Wesen der Sprache gelehrt haben. München. [Engl. Originalausg. New York 2012: Language: The cultural tool.]

George, Kristin (2014): Grammatische Techniken der Erinnerung. Was leisten grammatische Techniken zur Simulation von Erinnerung in Uwe Timms Am Beispiel meines Bruders? Masterarbeit. Universität Kassel.

Goody, Jack (1977): The domestication of the savage mind. (= Themes in the Social Sciences). Cambridge.

Goody, Jack (1987): The interface between the written and the oral. (= Studies in Literacy, Family, Culture and the State). Cambridge.

Günthner, Susanne (2008): Projektorkonstruktionen im Gespräch: Pseudoclefts, die Sache ist-Konstruktionen und Extrapositionen mit es. In: Gesprächsforschung. Online-Zeitschrift zur verbalen Interaktion 9, S. 86-114.

Günthner, Susanne/Hopper, Paul (2010): Zeitlichkeit und sprachliche Strukturen: Pseudoclefts im Englischen und im Deutschen. In: Gesprächsforschung. Online-Zeitschrift zur verbalen Interaktion 11, S. 1-18.

Härd, John Evert (1981): Studien zur Struktur mehrgliedriger deutscher Nebensatzprädikate. Diachronie und Synchronie. Göteborg.

Harris, Roy (1980): The language-makers. London.

Havelock, Eric. A. (1963): Preface to Plato. Cambridge, MA.

Hennig, Mathilde (2006): Grammatik der gesprochenen Sprache in Theorie und Praxis. Kassel. Hennig, Mathilde (2009): Nähe und Distanzierung. Verschriftlichung und Reorganisation des Nähebereichs im Neuhochdeutschen. Kassel. 
Hennig, Mathilde (2010a): Aggregative Koordinationsellipsen im Neuhochdeutschen. In: Ziegler (Hg.) (2010b), S. 937-963.

Hennig, Mathilde (2010b): Elliptische Junktion in der Syntax des Neuhochdeutschen. In: Schmid, Hans Ulrich (Hg.): Perspektiven der germanistischen Sprachgeschichtsforschung. (= Jahrbuch für germanistische Sprachgeschichte 1). Berlin/New York, S. 76-103.

Himmelmann, Nikolaus P. (1997): Deiktikon, Artikel, Nominalphrase. Zur Emergenz syntaktischer Struktur. (= Linguistische Arbeiten 362). Tübingen.

Hinterhölzl, Roland (2010): Zur Herausbildung der Satzklammer im Deutschen. Ein Plädoyer für eine informationsstrukturelle Analyse. In: Ziegler (Hg.) (2010a), S. 121-138.

Jaynes, Julian (1993): Der Ursprung des Bewußtseins. Reinbek bei Hamburg. [Engl. Originalausg. Boston 1976: The origin of consciousness in the breakdown of the bicameral mind.]

KAJUK = Kasseler Junktionskorpus. Internet: www.uni-giessen.de/kajuk/ (Stand 7.6.2014). Kappel, Péter (2014): Satzintegration in neuhochdeutschen Texten. Zum Schnittstellencharakter der Integration vorangestellter Adverbialsätze. In: Bassola, Péter et al. (Hg.): Zugänge zum Text. Frankfurt a.M. u.a., S. 193-215.

Koch, Peter (2010): Sprachgeschichte zwischen Nähe und Distanz: Latein - Französisch - Deutsch. In: Ágel, Vilmos/Hennig, Mathilde (Hg.): Nähe und Distanz im Kontext variationslinguistischer Forschung. (= Linguistik - Impulse \& Tendenzen 35). Berlin, S. 155-206.

Koch, Peter/Oesterreicher, Wulf (1994): Schriftlichkeit und Sprache. In: Günther, Hartmut/ Ludwig, Otto (Hg.): Schrift und Schriftlichkeit: ein interdisziplinäres Handbuch internationaler Forschung/Writing and its use. 1. Halbbd. (= Handbücher zur Sprach- und Kommunikationswissenschaft 10.1). Berlin/New York, S. 587-604.

Koch, Peter/Oesterreicher, Wulf (2011): Gesprochene Sprache in der Romania. Französisch, Italienisch, Spanisch. 2., aktual. u. erw. Aufl. (= Romanistische Arbeitshefte 31). Berlin/ New York.

Köller, Wilhelm (2004): Perspektivität und Sprache. Zur Struktur von Objektivierungsformen in Bildern, im Denken und in der Sprache. Berlin/New York.

Kotin, Michail L./Schönherr, Monika (2012): Zum Verhältnis von Epistemizität und Evidentialität im Deutschen aus diachroner und typologischer Sicht. In: Zeitschrift für deutsche Philologie 131, S. 393-416.

Linell, Per (2005): The written language bias in Linguistics: Its nature, origins and transformations. (= Routledge Advances in Communication and Linguistic Theory 5). London.

Lötscher, Andreas (1995): Herausstellung nach links in diachroner Sicht. In: Sprachwissenschaft 20, S. 32-63.

Maas, Utz (2010): Literat und orat. Grundbegriffe der Analyse geschriebener und gesprochener Sprache. In: Grazer Linguistische Studien 73, S. 21-150.

Malinowski, Bronislaw (1974): Das Problem der Bedeutung in primitiven Sprachen. In: Ogden, Charles K./Richards, Ivor A.: Die Bedeutung der Bedeutung. Eine Untersuchung über den Einfluß der Sprache auf das Denken und über die Wissenschaft des Symbolismus. Supplement. Frankfurt a.M., S. 323-384. [Engl. Originalausg. New York 1923: The problem of meaning in primitive languages.]

Mánássy, Ildikó (2005): Arten der Disgruenz. Disgruenzfördernde Faktoren in historischen Texten. Abschlussarbeit. Universität Szeged.

Ong, Walter J. (1987): Oralität und Literalität. Die Technologisierung des Wortes. Opladen. [Engl. Originalausg. New York 1982: Orality and literacy: The technologizing of the word.] 
Näf, Anton (1979): Die Wortstellung in Notkers Consolatio. Untersuchungen zur Syntax und Übersetzungstechnik. Berlin/New York.

Parente, Maria Alice de Mattos Pimenta/Fonseca, Rochele Paz/Scherer, Lilian Cristine (2008): Literacy as a determining factor for brain organization: from Lecours' contribution to the present day. In: Dementia \& Neuropsychologia 2, S. 165-172.

Raible, Wolfgang (1992): Junktion. Eine Dimension der Sprache und ihre Realisierungsformen zwischen Aggregation und Integration. (= Sitzungsberichte der Heidelberger Akademie der Wissenschaften, Philosophisch-historische Klasse 1992/2). Heidelberg.

Reichmann, Oskar (1988): Zur Vertikalisierung des Varietätenspektrums in der jüngeren Sprachgeschichte des Deutschen. In: Munske, Horst Haider et al. (Hg.): Deutscher Wortschatz. Lexikologische Studien. Festschrift für Ludwig Erich Schmitt von seinen Marburger Schülern. Berlin/New York, S. 151-180.

Reichmann, Oskar (1990): Sprache ohne Leitvarietät vs. Sprache mit Leitvarietät: ein Schlüssel für die nachmittelalterliche Geschichte des Deutschen? In: Besch, Werner (Hg.): Deutsche Sprachgeschichte. Grundlagen, Methoden, Perspektiven. Festschrift für Johannes Erben zum 65. Geburtstag. Frankfurt a.M. u.a., S. 141-158.

Reichmann, Oskar (1995): Die Konzepte von ,Deutlichkeit“ und ,Eindeutigkeit‘ in der rationalistischen Sprachtheorie des 18. Jahrhunderts. In: Gardt, Andreas/Mattheier, Klaus J./ Reichmann, Oskar (Hg.): Sprachgeschichte des Neuhochdeutschen. Gegenstände, Methoden, Theorien. (= Reihe Germanistische Linguistik 156). Tübingen, S. 169-197.

Reichmann, Oskar (2003): Die Entstehung der neuhochdeutschen Schriftsprache: Wo bleiben die Regionen? In: Berthele, Raphael et al. (Hg.): Die deutsche Schriftsprache und die Regionen. Entstehungsgeschichtliche Fragen in neuer Sicht. (= Studia Linguistica Germanica 65). Berlin/New York, S. 29-56.

Reis, Marga (1980): On justifying topological frames. ,Positional Field“ and the order of nonverbal constituents in German. In: DRLAV: Revue de Linguistique 22/23, S. 59-85.

Sampson, Geoffrey/Babarczy, Anna (2014): Grammar without grammaticality. Growth and limits of grammatical precision. (= Trends in Linguistics. Studies and Monographs 254). Berlin/ Boston.

Schallert, Oliver (2010): Als Deutsch noch nicht OV war. Althochdeutsch im Spannungsfeld zwischen OV und VO. In: Ziegler (Hg.) (2010a), S. 365-394.

Scheerer, Eckart (1993): Mündlichkeit und Schriftlichkeit - Implikationen für die Modellierung kognitiver Prozesse. In: Baurmann, Jürgen/Günther, Hartmut/Knoop, Ulrich (Hg.): homo scribens. Perspektiven der Schriftlichkeitsforschung. (= Reihe Germanistische Linguistik 134). Tübingen, S. 141-176.

Schlachter, Eva (2010): Zum Verhältnis von Stil und Syntax. Die Verbfrüherstellung in Zitat- und Traktatsyntax des althochdeutschen Isidor. In: Ziegler (Hg.) (2010a), S. 409-425.

Schmidt, Jürgen Erich (1993): Die deutsche Substantivgruppe und die Attribuierungskomplikation. (= Reihe Germanistische Linguistik 138). Tübingen.

Schmidt, Jürgen Erich/Herrgen, Joachim (2011): Sprachdynamik: Eine Einführung in die moderne Regionalsprachenforschung. (= Grundlagen der Germanistik 49). Berlin.

Schneider, Jan Georg (2011): Hat die gesprochene Sprache eine eigene Grammatik? Grundsätzliche Überlegungen zum Status gesprochensprachlicher Konstruktionen und zur Kategorie, gesprochenes Standarddeutsch‘. In: Zeitschrift für Germanistische Linguistik 39, S. 165-187.

Schrodt, Richard (2004): Althochdeutsche Grammatik. Bd. 2: Syntax. (= Sammlung kurzer Grammatiken germanischer Dialekte $A / 5,2$ ). Tübingen. 
Selting, Margret (1995): Prosodie im Gespräch. Aspekte einer interaktionalen Phonologie der Konversation. (= Linguistische Arbeiten 329). Tübingen.

Van Lancker, Diana (1987): Nonpropositional speech: Neurolinguistic studies. In: Ellis, Andrew W. (Hg.): Progress in the psychology of language. Bd. 3. London, S. 49-118.

Wöllstein, Angelika (2010): Topologisches Satzmodell. (= Kurze Einführungen in die Germanistische Linguistik 8). Heidelberg.

Zeman, Sonja (2013): Historische Mündlichkeit. Empirische Erörterung einer theoretischen Problemlage. In: Zeitschrift für Germanistische Linguistik 41, S. 377-412.

Ziegler, Arne (Hg.) (2010a): Historische Textgrammatik und Historische Syntax des Deutschen. Traditionen, Innovationen, Perspektiven. Bd. 1: Diachronie, Althochdeutsch, Mittelhochdeutsch. Unter Mitarbeit v. Christian Braun. Berlin.

Ziegler, Arne (Hg.) (2010b): Historische Textgrammatik und Historische Syntax des Deutschen. Traditionen, Innovationen, Perspektiven. Bd. 2: Frühneuhochdeutsch, Neuhochdeutsch. Unter Mitarbeit v. Christian Braun. Berlin

Zwicky, Arnold M. (1993): Heads, bases and functors. In: Corbett, Greville G./Fraser, Norman M./McGlashan, Scott (Hg.): Heads in grammatical theory. Cambridge, S. 292-315. 